\title{
SMASH: Survey of the MAgellanic Stellar History
}

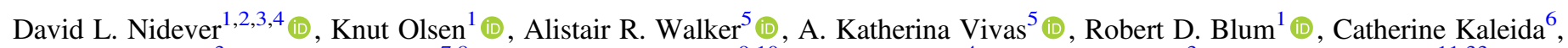

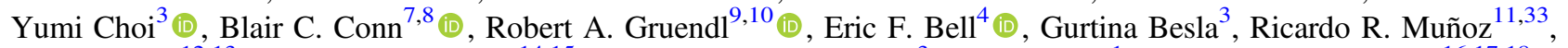
Carme Gallart ${ }^{12,13}$ (1) Nicolas F. Martin ${ }^{14,15}$ (1) Edward W. Olszewski ${ }^{3}$, Abhijit Saha ${ }^{1}$ (i), Antonela Monachesi ${ }^{16,17,18}$ (1), Matteo Monelli ${ }^{12,13}$ (1) Thomas J. L. de Boer ${ }^{19}$, L. Clifton Johnson ${ }^{20}$ (1) , Dennis Zaritsky ${ }^{3}$ (), Guy S. Stringfellow ${ }^{21}$ (i),

Roeland P. van der Marel ${ }^{6}$ (10), Maria-Rosa L. Cioni ${ }^{22,23,24}$ (10), Shoko Jin ${ }^{25}$ (D), Steven R. Majewski ${ }^{26}$ (i),

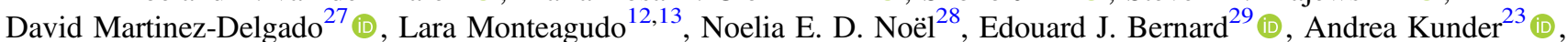

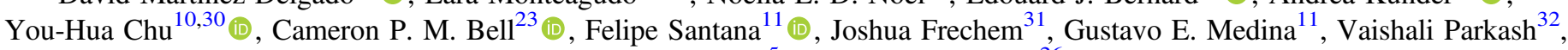
J. C. Serón Navarrete ${ }^{5}$, and Christian Hayes ${ }^{26}$

${ }^{1}$ National Optical Astronomy Observatory, 950 North Cherry Avenue, Tucson, AZ 85719, USA; dnidever@noao.edu

${ }^{2}$ Large Synoptic Survey Telescope, 950 North Cherry Avenue, Tucson, AZ 85719, USA

${ }^{3}$ Steward Observatory, University of Arizona, 933 North Cherry Avenue, Tucson AZ, 85721, USA

${ }_{5}^{4}$ Department of Astronomy, University of Michigan, 1085 S. University Avenue, Ann Arbor, MI 48109-1107, USA

${ }^{5}$ Cerro Tololo Inter-American Observatory, National Optical Astronomy Observatory, Casilla 603, La Serena, Chile

${ }^{6}$ Space Telescope Science Institute, 3700 San Martin Drive, Baltimore, MD 21218, USA

${ }^{7}$ Research School of Astronomy and Astrophysics, Australian National University, Canberra, ACT 2611, Australia

${ }^{8}$ Gemini Observatory, Recinto AURA, Colina El Pino s/n, La Serena, Chile

${ }^{9}$ National Center for Supercomputing Applications, 1205 West Clark Street, Urbana, IL 61801, USA

${ }^{10}$ Department of Astronomy, University of Illinois, 1002 West Green Street, Urbana, IL 61801, USA

${ }^{11}$ Departamento de Astronomía, Universidad de Chile, Camino del Observatorio 1515, Las Condes, Santiago, Chile

${ }^{12}$ Instituto de Astrofísica de Canarias, La Laguna, Tenerife, Spain

${ }^{13}$ Departamento de Astrofísica, Universidad de La Laguna, Tenerife, Spain

${ }^{14}$ Université de Strasbourg, CNRS, Observatoire astronomique de Strasbourg, UMR 7550, F-67000 Strasbourg, France

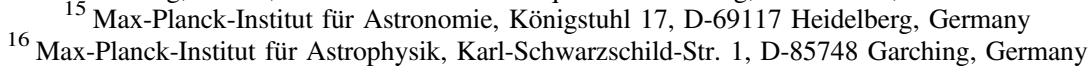

${ }^{17}$ Instituto de Investigación Multidisciplinario en Ciencia y Tecnología, Universidad de La Serena, Raúl Bitrán 1305, La Serena, Chile

${ }^{18}$ Departamento de Física y Astronomía, Universidad de La Serena, Av. Juan Cisternas 1200 N, La Serena, Chile

${ }^{19}$ Institute of Astronomy, University of Cambridge, Madingley Road, Cambridge CB3 OHA, UK

${ }^{20}$ Center for Astrophysics and Space Sciences, UC San Diego, 9500 Gilman Drive, La Jolla, CA, 92093-0424, USA

${ }_{21}^{21}$ Center for Astrophysics and Space Astronomy, University of Colorado, 389 UCB, Boulder, CO, 80309-0389, USA

${ }^{22}$ Universität Potsdam, Institut für Physik und Astronomie, Karl-Liebknecht-Str. 24/25, D-14476 Potsdam, Germany

${ }^{23}$ Leibniz-Institut für Astrophysics Potsdam (AIP), An der Sternwarte 16, D-14482 Potsdam Germany

${ }^{24}$ University of Hertfordshire, Physics Astronomy and Mathematics, Hatfield AL10 9AB, UK

${ }^{25}$ STFC RALSpace, Rutherford Appleton Laboratory, Harrell Oxford OX11 OQX UK

${ }^{26}$ Department of Astronomy, University of Virginia, Charlottesville, VA 22904, USA

${ }^{27}$ Astronomisches Rechen-Institut, Zentrum für Astronomie der Universität Heidelberg, Mönchhofstr. 12-14, D-69120 Heidelberg, Germany

${ }^{2}$ Department of Physics, University of Surrey, Guildford, GU2 7XH, UK

${ }^{29}$ Université Côte d'Azur, OCA, CNRS, Lagrange, France

${ }^{30}$ Institute of Astronomy and Astrophysics, Academia Sinica, No.1, Sec. 4, Roosevelt Road, Taipei 10617, Taiwan, R.O.C.

${ }^{31}$ Rochester Institute of Technology, Rochester, NY 14623, USA

${ }^{32}$ Monash Centre for Astrophysics, School of Physics and Astronomy, Monash University, Victoria 3800, Australia

Received 2017 January 2; revised 2017 September 12; accepted 2017 September 13; published 2017 October 25

\begin{abstract}
The Large and Small Magellanic Clouds are unique local laboratories for studying the formation and evolution of small galaxies in exquisite detail. The Survey of the MAgellanic Stellar History (SMASH) is an NOAO community Dark Energy Camera (DECam) survey of the Clouds mapping $480 \mathrm{deg}^{2}$ (distributed over $\sim 2400$ square degrees at $\sim 20 \%$ filling factor) to $\sim 24$ th mag in ugriz. The primary goals of SMASH are to identify low surface brightness stellar populations associated with the stellar halos and tidal debris of the Clouds, and to derive spatially resolved star formation histories. Here, we present a summary of the survey, its data reduction, and a description of the first public Data Release (DR1). The SMASH DECam data have been reduced with a combination of the NOAO Community Pipeline, the PHOTRED automated point-spread-function photometry pipeline, and custom calibration software. The astrometric precision is $\sim 15$ mas and the accuracy is $\sim 2$ mas with respect to the Gaia reference frame. The photometric precision is $\sim 0.5 \%-0.7 \%$ in griz and $\sim 1 \%$ in $u$ with a calibration accuracy of $\sim 1.3 \%$ in all bands. The median $5 \sigma$ point source depths in ugriz are 23.9, 24.8, 24.5, 24.2, and $23.5 \mathrm{mag}$. The SMASH data have already been used to discover the Hydra II Milky Way satellite, the SMASH 1 old globular cluster likely associated with the LMC, and extended stellar populations around the LMC out to $R \sim 18.4 \mathrm{kpc}$. SMASH DR1 contains measurements of $\sim 100$ million objects distributed in 61 fields. A prototype version of the NOAO Data Lab provides data access and exploration tools.
\end{abstract}

\footnotetext{
${ }^{33}$ Visiting astronomer, Cerro Tololo Inter-American Observatory, National Optical Astronomy Observatory, which is operated by the Association of Universities for Research in Astronomy (AURA) under a cooperative agreement with the National Science Foundation.
} 
Key words: galaxies: dwarf - galaxies: individual (Large Magellanic Cloud, Small Magellanic Cloud) Local Group - Magellanic Clouds - surveys

Supporting material: machine-readable table

\section{Introduction}

The Large and Small Magellanic Clouds (LMC and SMC), as two of the nearest and most massive satellite galaxies of the Milky Way (MW), offer a unique opportunity to study the processes of galaxy formation and evolution of low-mass galaxies in great detail. The Clouds have long held broad importance for astronomy, both as laboratories of astrophysical processes and as calibrators of the extragalactic distance scale. As the closest example of an interacting pair of galaxies, they provide special insight into the impact of such interactions on the structure and evolution of galaxies.

The Clouds are ideally suited to addressing some particularly critical questions: What are the consequences of the stripping of stars and gas when dwarf galaxies fall into the halos of more massive galaxies, an important mode of mass growth for galaxies since $z \sim 1$ ? What are the properties of the hot and warm gaseous halos of galaxies like the MW, the density of which sets the efficiency of gas stripping and "quenching" of satellites? What are the physical mechanisms and timescales, if any, behind the triggering of star formation by galaxy interactions? These questions can only be addressed by surveys that probe the stars, gas, and dust of the Magellanic Clouds (MCs) and their surroundings.

de Vaucouleurs (1955) began the systematic study of the MCs, highlighting the young and bright structures that trace the LMC's bar and the SMC's very irregular shape. Although the gaseous component of the LMC's disk extends only to a radius of $\sim 4^{\circ}$ (Staveley-Smith et al. 2003), the stellar component stretches over a much larger area. Stellar catalogs from the large near-infrared surveys DENIS (Epchtein et al. 1997) and 2MASS (Skrutskie et al. 2006) show it extending to at least $\sim 8^{\circ}$ and were used to measure the LMC disk structure and viewing angle (van der Marel 2001; van der Marel \& Cioni 2001). Furthermore, carbon stars from Kunkel et al. (1997) follow disk kinematics out to $\sim 13^{\circ}$ (van der Marel et al. 2002). Finally, the Outer Limits Survey (OLS; Saha et al. 2010) used deep photometry to study old LMC main-sequence stars in select fields far from the LMC center and found that in the north they followed the disk exponential profile out to $\sim 16^{\circ}$.

Indeed, the peripheries of the MCs have been a rich ground for new discoveries. In the LMC, Minniti et al. (2003) found kinematical evidence for an old stellar halo using RR Lyrae stars (in the central region) with a large velocity dispersion. In the LMC outskirts, Muñoz et al. (2006) discovered a kinematically cold group of LMC stars in the foreground of the Carina dwarf spheroidal galaxy. Subsequently, the MAgellanic Periphery Survey (MAPS; described in Nidever et al. 2011) used spectroscopically confirmed red giant branch stars to detect a halo-like stellar population extending out to $\sim 22^{\circ}$ (over an $\sim 180^{\circ}$ azimuthal range) following a shallow de Vaucouleurs profile (Majewski et al. 2009). More recently, Mackey et al. (2016) used Dark Energy Survey (DES; Dark Energy Survey Collaboration et al. 2016) data to detect an "arclike" structure in the periphery of the LMC $\left(\sim 15^{\circ}\right.$ from the center), which is likely a tidally disturbed portion of the LMC disk due to a recent interaction with the SMC. In addition, Belokurov et al. (2017) used DES data to map streams of Blue
Horizontal Branch (BHB) stars around the MCs with some extending to $R \sim 40^{\circ}$.

In the SMC, early photometric plate photometry showed that the young blue stars (produced by recent intense star formation) have a very irregular and patchy distribution while the older population (traced by giant stars) is fairly azimuthally symmetric and extends to larger radii $\left(\sim 5^{\circ}\right.$; Hatzidimitriou \& Hawkins 1989; Gardiner \& Hawkins 1991; Gardiner \& Hatzidimitriou 1992), as expected for dIrr galaxies (Mateo 1998). Subsequent deep CCD photometry by Noël \& Gallart (2007) found old stellar populations out 5.8 and De Propris et al. (2010) detected red giant stars spectroscopically out to $\sim 6^{\circ}$. The MAPS survey used photometrically selected red giant stars to find that the older SMC stellar population followed the same exponential profile from the center of the SMC out to $\sim 7^{\circ} .5$, but that beyond that there was a break in the radial profile with stars extending out to $\sim 11^{\circ}$ (later confirmed with DES data by Pieres et al. 2017), potentially representing a classical stellar halo of the SMC. In addition, Nidever et al. (2013) found evidence for a stellar component of the tidally stripped Magellanic Bridge; more recently, Belokurov \& Koposov (2016) used Gaia DR1 data to find both young and old stellar bridges, although they are not spatially coincident, between the Clouds. Finally, Pieres et al. (2017) used DES data to discover a stellar overdensity in the northern region of the SMC (at $\sim 8^{\circ}$ ) that might be a tidally stripped dwarf galaxy.

Explanations for the structure found in the periphery of the MCs must naturally account for the complex history of interaction that they have had with each other and the MW. These interactions, which have produced the gaseous Magellanic Stream, Leading Arm, and Bridge (Stanimirović et al. 1999; Muller et al. 2003; Putman et al. 2003; Brüns et al. 2005; Nidever et al. 2008, 2010), have been explored for over three decades (e.g., Murai \& Fujimoto 1980; Gardiner \& Noguchi 1996; Yoshizawa \& Noguchi 2003; Connors et al. 2004, 2006; Mastropietro et al. 2005; Besla et al. 2012, 2013). Many of the features of the Magellanic system have been well-reproduced by a model invoking tidal stripping through repeated close passages to the MW by the MCs on their bound orbit (e.g., Gardiner \& Noguchi 1996), although ram pressure stripping (e.g., Mastropietro et al. 2005) and star formation feedback (e.g., Olano 2004; Nidever et al. 2008) have also been put forward as origin mechanisms and are likely important factors. However, recent HST-based proper motion measurements of the MCs (Kallivayalil et al. 2006a, 2006b, 2013) indicate that the MCs have higher velocities relative to the MW than previously thought. Such high velocities may allow for multiple interactions of the Clouds with the MW (Bekki 2011; Gómez et al. 2015). A more likely scenario, however, is that the MCs are approaching the MW environment for the first time (Besla et al. 2007; Gómez et al. 2015), as favored by cosmological simulations (Boylan-Kolchin et al. 2011; Busha et al. 2011; Patel et al. 2017). This discovery has forced a reinterpretation of many features of the Magellanic System, leading recent simulations (Besla et al. 2010, 2012; Diaz \& Bekki 2012) to suggest that LMC-SMC interactions alone are responsible for the formation of the Magellanic Stream, 
including a potential direct collision between the LMC and SMC. The discovery that the LMC has stripped a large number of stars from the SMC ( $\sim 5 \%$ of the LMC's mass; Olsen et al. 2011) supports the collision hypothesis, or at least necessitates a very close interaction between the SMC and LMC. If that collision also produced the Stream and Leading Arm, which are now known to extend for at least $200^{\circ}$ across the sky (Nidever et al. 2010), then there ought to be stellar tidal debris over a similar area of sky as well, with potentially high densities in the area of the Leading Arm (Besla et al. 2010).

The primary aim of the Survey of the MAgellanic Stellar History (SMASH) is to trace and to measure the extended stellar populations of the Clouds, including potential tidal debris, extended disks, and halo components. SMASH is an NOAO survey project that is using the Dark Energy Camera (DECam; Flaugher et al. 2015) on the NOAO Blanco $4 \mathrm{~m}$ telescope at Cerro Tololo Inter-American Observatory (CTIO) to observe a total area of $480 \mathrm{deg}^{2}$ of the Magellanic periphery and the main bodies with deep ugriz images. SMASH fields completely cover the main bodies of the Clouds, while in the periphery they are distributed as "islands" over an area of $\sim 2400 \mathrm{deg}^{2}$ to maximize the chances of detecting widely distributed stellar populations within the allotted observing time. SMASH builds on the technique first adopted by the OLS (Saha et al. 2010), using old main-sequence stars as tracers of populations down to surface brightnesses equivalent to $\Sigma_{g}=35 \mathrm{mag} \operatorname{arcsec}^{-2}$. Compared to the $\sim 5000 \mathrm{deg}^{2}$ DES, SMASH covers an order of magnitude less total area, but probes a region that is only a factor of two smaller in size. Whereas DES covers the northern MC periphery and includes the Magellanic Stream, SMASH covers the southern periphery (as well as the MC main bodies) and includes the Leading Arm, where models by Besla et al. (2010) predict that tidal debris is prominent. SMASH photometry is as deep or deeper than DES in griz and includes the $u$ filter, which DES does not have, but does not include the $Y$ filter.

The SMASH survey addresses a number of important scientific goals, progress for which is underway. The detection of stellar debris in either the Leading Arm or Magellanic Stream would make them the only tidal streams with known gaseous and stellar components in the Local Group. This would not only be invaluable for understanding the history and observable consequences of the Magellanic interaction, but would give us a "clean" dynamical tracer of the MW's dark halo and a way to prove the efficiency of the MW's hot halo gas to induce ram pressure effects. The size of the LMC's stellar periphery is a direct probe of the tidal radius of the LMC, with which we can explore the dark matter halos of both the LMC and MW. DES has led to the discovery of many new satellite galaxies (Bechtol et al. 2015; Drlica-Wagner et al. 2015; Koposov et al. 2015), some of which are likely associated with the MCs (Deason et al. 2015b; Jethwa et al. 2016; Walker et al. 2016; Sales et al. 2017). The discovery that satellite galaxies may have their own satellite systems is an important new piece to the puzzle of the "missing satellites" (Klypin et al. 1999; Moore et al. 1999). We have discovered one new satellite galaxy in SMASH, Hydra II (Martin et al. 2015), which, from its position and distance, may be associated with the Leading Arm of the Magellanic Stream; the search for more is in progress. Finally, it is known that strong population gradients exist to large radii in both MCs (Gallart et al. 2008; Cioni 2009). With SMASH, we are working to derive spatially resolved, precise star formation histories covering all ages of the MCs and to the largest radii, thus providing detailed information on their complete evolution. We are also working to identify new star clusters and to map the MC's galactic structures.

Besides SMASH, there are other optical and NIR surveys of the MCs. The fourth phase of the Optical Gravitational Lensing Experiment (OGLE-IV; Udalski et al. 2015) is contiguously covering the main bodies of the MCs as well as the Bridge region between them with relatively shallow $V I(\sim 21 \mathrm{mag})$ photometry but with many repeat observations that make it useful for studying the distribution of Magellanic variable stars (Skowron et al. 2014; Jacyszyn-Dobrzeniecka et al. 2016, 2017; Soszyński et al. 2016). The VISTA survey of the Magellanic Clouds system (VMC; Cioni et al. 2011) is a nearinfrared $Y J K_{s}$ survey of the main bodies of the MCs that, among other things, is being used to study the star formation history (Rubele et al. 2015) and proper motion (Cioni et al. 2016) of the Clouds. In addition, the STEP survey using the VST telescope is an optical gi-band ( $\sim 23.5 \mathrm{mag})$ survey of the SMC and Bridge region with repeat observations (Ripepi et al. 2014) As previously mentioned, the contiguous DES survey serendipitously covers the northern regions of the MCs as well as the Magellanic Stream and has been used to study the Magellanic system (Belokurov \& Koposov 2016; Mackey et al. 2016). Finally, the Magellanic Satellites Survey (MagLiteS; described in Drlica-Wagner et al. 2016) is searching for Magellanic satellites using shallow $g$ and $r$ DECam images in a $\sim 1200 \mathrm{deg}^{2}$ region around the southern periphery of the MCs.

Although scientific analysis with SMASH data have been published and are underway, we expect that there are many projects that others will think of and do first, as evidenced by the discovery of new star cluster candidates in the MCs by Piatti (2017) using SMASH data. The aim of this paper is to enable more of such projects by describing the SMASH survey, providing details on the pipelines that produced the photometric catalogs, and providing information on the first public data release. The layout of this paper is as follows. Sections 2 and 3 detail the survey and observing strategy. An overview of the image processing is given in Section 4 , the photometric reduction is given in Section 5, while the calibration is discussed in Section 6. A description of the final catalogs and the achieved performance is given in Section 7. The details of the first SMASH data release and data access are described in Section 8, and finally, some of the first SMASH science results are discussed in Section 9.

\section{Survey Strategy}

Figure 1 shows the region of the sky that is relevant to the MCs and the Magellanic Stream, ${ }^{34}$ with the H I distribution in the top panel and the predicted stellar distribution of the Besla et al. (2013) model in the bottom panel. The DES footprint already covers one-half of the LMC/SMC periphery as well as much of the trailing Magellanic Stream. The SMASH footprint was designed to cover the rest of the Magellanic periphery and the Leading Arm, ${ }^{35}$ but to avoid the MW midplane that could "contaminate" the data. A fully filled survey would have been

\footnotetext{
34 Also see Figure 10, which has more information on the SMASH fields including field names and calibration status.

${ }^{35}$ Note that even though the SMASH fields were designed to be complementary to the DES survey, the DES footprint changed over the last couple of years, producing some overlap and gaps between the two surveys.
} 


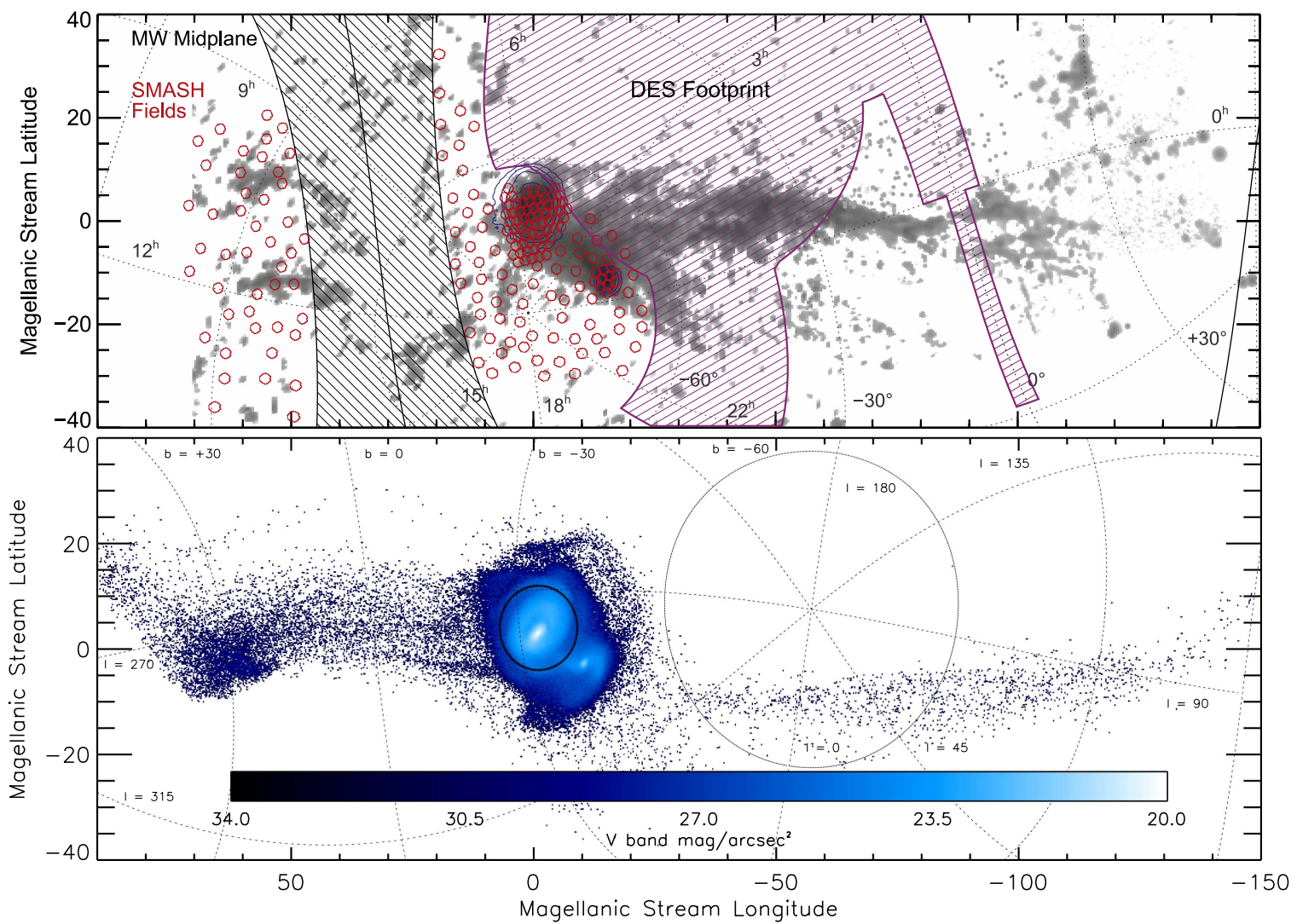

Figure 1. Region of the sky relevant to the Magellanic system in the Magellanic Stream coordinates system (Nidever et al. 2008). (Top) The observed H I column density of the entire $200^{\circ}$ Magellanic Stream system (Nidever et al. 2010) is shown in grayscale, while the blue contours represent the 2MASS (Skrutskie et al. 2006) red giant branch star counts. The open red hexagons are the SMASH survey fields (with the correct size and shape). The DES footprint is represented by the purple shaded region. The solid line represents the Galactic equator, the hashed region is a Galactic Zone of Avoidance region, and the dotted lines are an equatorial coordinate grid. (Bottom) The predicted $V$-band surface brightness (mag arcsec ${ }^{-2}$ ) of the stellar component of the Magellanic system from Besla et al. (2013). The simulation predicts stellar structures out to large radii from the main bodies of the MCs (varying on small scales) and a higher stellar density in the Leading Arm than in the trailing Stream. In the absence of strong gas drag, the stellar and gas components are expected to be coincident. In this model, the exact location of the debris is not tuned to match the observations, but the relative surface brightness of the various components is a robust prediction. A Galactic coordinate grid is shown by the dotted lines.

preferred, but to reach the sensitivity required to detect the predicted low surface brightness features would have required hundreds of nights and would be beyond the possibility of an NOAO survey proposal. We, therefore, decided to pursue a deep but partially filled survey strategy as is shown in the top of Figure 1 (red hexagons). The SMASH fields map an area of $\sim 480 \mathrm{deg}^{2}$ but are distributed over (and probe the stellar populations of) $\sim 2400 \mathrm{deg}^{2}$ with a filling factor of $\sim 20 \%$.

The DECam imager is composed of 62 chips ( 59 and a half were functional throughout most of the SMASH observing; CCDNUM 2, 61, and one amplifier of 31 were not producing useful science data, although CCDNUM 2 started working again as of 2016 December 29) from Lawrence Berkeley National Lab (LBNL) arranged in a hexagonal pattern covering a field of view of $\sim 3 \mathrm{deg}^{2}$ and a width of $\sim 2^{\circ}$. The SMASH fields were chosen using an all-sky tiling scheme in which we laid down a uniform hex pattern of field centers with 1.7 separation between field centers with coordinates based on an Interrupted Mollweide projection. This spherical projection has low distortion, such that uniform sampling in its coordinate system produces tiling with few areas of excessive overlap between fields. We then transformed the coordinates of the hex-based tiling to spherical coordinates and rotated the coordinate system to place the seams and poles (southern pole of $[\alpha, \delta]=\left[10^{\circ},-30^{\circ}\right]$ ) in areas outside of our survey area.
The resulting tiling of the sky was nearly uniform over our survey area with $\sim 15 \%$ areal overlap between neighboring fields to allow for good cross-calibration (although this was only used in our main-body fields).

From this list of tiles, we selected 154 fields by hand to uniformly sample the region of interest with a 20\% filling factor as well as fully cover the inner regions of the LMC and SMC. The full coverage tiling scheme and overlap were used so that, given more observing opportunities, we could more easily completely cover regions with interesting stellar populations later on, which is what we did for the outer LMC disk. Note that the final survey tiling scheme was created after the 2013 March 17-20 pre-survey run. Therefore, the positions of the 23 Leading Arm fields (Fields 153-175) that were observed on that run are not entirely consistent with those of the final tiling scheme, but the differences are not significant. The final list of SMASH fields with coordinates in various systems is available in the machine-readable version of Table 1.

\section{Observing Strategy and Observations}

The idea for SMASH was conceived during the NOAO "Seeing the Big Picture: DECam Community Workshop" in Tucson, AZ, on 2011 August 18-19. We decided to submit a proposal for an MC pilot project using Science Verification (SV) and Shared Risk (SR) time during the 2012B season. The 
Table 1

SMASH Fields Table

\begin{tabular}{|c|c|c|c|c|c|c|c|c|c|c|c|c|}
\hline Number & Name & RAJ2000 & DEJ2000 & RADEG & DEDEG & $L_{\mathrm{MS}}{ }^{\mathrm{a}}$ & $B_{\mathrm{MS}}{ }^{\mathrm{a}}$ & uZPCal & gZPCal & rZPCal & iZPCal & $\overline{\mathrm{zZPCal}}$ \\
\hline 1 & 0010-6947 & $00: 10: 19.87$ & $-69: 47: 40.56$ & 2.58282 & -69.794600 & -19.56937 & -13.84173 & 4 & 4 & 4 & 4 & 4 \\
\hline 2 & 0018-7705 & 00:18:57.90 & $-77: 05: 00.23$ & 4.74128 & -77.083400 & -12.11547 & -15.01799 & 4 & 4 & 4 & 4 & 4 \\
\hline 3 & $0023-7358$ & $00: 23: 19.08$ & $-73: 58: 08.04$ & 5.82950 & -73.968900 & -15.14808 & -13.94466 & 1 & 1 & 1 & 1 & 1 \\
\hline 4 & $0024-7223$ & $00: 24: 56.64$ & $-72: 23: 08.15$ & 6.23604 & -72.385600 & -16.67737 & -13.38564 & 1 & 1 & 1 & 1 & 1 \\
\hline 5 & 0044-7137 & 00:44:06.76 & $-71: 37: 45.84$ & 11.02820 & -71.629400 & -16.91433 & -11.74021 & 1 & 1 & 1 & 1 & 1 \\
\hline 6 & 0044-7313 & $00: 44: 32.54$ & $-73: 13: 31.79$ & 11.13560 & -73.225500 & -15.38135 & -12.28828 & 1 & 1 & 1 & 1 & 1 \\
\hline 7 & $0045-7448$ & $00: 45: 03.28$ & $-74: 48: 14.76$ & 11.26370 & -74.804100 & -13.85882 & -12.82186 & 1 & 1 & 1 & 1 & 1 \\
\hline 8 & 0050-8228 & $00: 50: 34.51$ & $-82: 28: 26.40$ & 12.64380 & -82.474000 & -6.36406 & -15.28088 & 1 & 1 & 1 & 1 & 1 \\
\hline 9 & 0101-7043 & 01:01:27.40 & $-70: 43: 05.51$ & 15.36420 & -70.718200 & -17.19874 & -10.09455 & 1 & 1 & 1 & 1 & 1 \\
\hline 10 & $0103-7218$ & 01:03:36.31 & $-72: 18: 54.36$ & 15.90130 & -72.315100 & -15.66017 & -10.63165 & 2 & 2 & 2 & 2 & 2 \\
\hline
\end{tabular}

Note. The full table is available in a machine-readable version and in the data/smash_fields_final.txt file on the SMASHRED repository (https://github.com/dnidever/SMASHRED).

${ }^{a}$ Magellanic Stream coordinates defined in Nidever et al. (2008).

(This table is available in its entirety in machine-readable form.) 
goal of the successful project (SV:2012B-3005 and SR:20120416) was to ascertain the necessary filters and depth to attain the needed sensitivity to Magellanic stellar populations. Data were obtained in five fields at various distances from the MCs and included exposures in all five ugriz bands and to a depth $\sim 1$ mag deeper than we thought was necessary for our science goals. These data helped us evaluate various observing and survey strategies. Ultimately, it was determined that all five bands would give us the sensitivity needed to detect the predicted stellar populations in the Magellanic periphery and the Leading Arm. In addition, the $u$-band would allow us to determine photometric metallicities for the Magellanic mainsequence stars (e.g., Ivezić et al. 2008), which would be useful in determining the origin of any newly found stellar populations.

After the pilot project, there was no call for survey proposals, so we proceeded to submit a regular NOAO proposal to look for stellar populations in the area of the Leading Arm (2013A0411). To maximize the coverage, we did not take $u$-band exposures for this observing run; however, the $u$-band exposures were obtained on later observing runs.

There was a call for survey proposals during the next semester, and we submitted a successful proposal for the SMASH survey of the MC stellar populations (2013B-0440). We were originally awarded 30 DECam nights (with a 7/3 A/B semester split) and fourteen $0.9 \mathrm{~m}$ nights for calibration purposes over three years. The standard SMASH observing sequence for a science field is three $60 \mathrm{~s}$ exposures (with large, half-chip offsets) in each band and three deep exposures with exposure times of $333 \mathrm{~s}(u), 267 \mathrm{~s}(g), 267 \mathrm{~s}(r), 333 \mathrm{~s}(i)$, and $333 \mathrm{~s}(z)$ with small $\sim 2^{\prime \prime}$ dithers. Each field takes about 110 minutes to observe, including readout time and slewing. Each night, exposures of four to five standard star fields (focusing on the York et al. 2000 SDSS equatorial region where data for all chips could be obtained simultaneously) were obtained with exposure times of $1 \mathrm{~s}$ in all ugriz bands as well as $10 \mathrm{~s}$ in griz and $60 \mathrm{~s}$ in $u$, but to save time, this was later changed (halfway through the survey) to single exposures of $15 \mathrm{~s}$ in griz and $60 \mathrm{~s}$ in $u$.

Due to bad weather, poor seeing (we have seeing constraints of $\lesssim 1^{\prime \prime}$ for the central LMC/SMC main-body fields because of crowding), and the short B semester nights, the survey fell behind in the MC main-body regions. Therefore, after the first year, we requested our 10 nights per year be split evenly between the A and B semesters (instead of 7/3 as before), and after the second year, we requested an additional three nights per semester in 2015B and 2016A. After our last year, we requested a three-night extension in 2016B to fill a "hole" in our coverage of the SMC periphery (near the south celestial pole) of 11 fields. Additional DECam nights were obtained through the Chilean TAC (PI: Muñoz; 2014 January 21-28). Finally, after the discovery of the Hydra II MW satellite in the SMASH data (Martin et al. 2015), we submitted a Director's Discretionary Time proposal to obtain time-series data on Hydra II to study variable stars (2015 March 30-31).

On our very successful 2016 February 13-18 run, we finished all of the fields around the MCs that were observable, and therefore, we decided to observe some "extra" shallow fields around the LMC that would help reveal structures in the LMC disk (similar to those seen by Mackey et al. 2016 and Besla et al. 2016) and allow us to use the field overlaps to create a more homogeneously calibrated data set around the
LMC using an "übercal" technique (e.g., Padmanabhan et al. 2008). Two $60 \mathrm{~s}$ exposures in griz with a half-chip dither between the pairs were obtained for these 40 fields (with field numbers between 184 and 243). The last SMASH observing run in the Leading Arm region (2016 May 8-12) was completely lost due to bad weather, and therefore, the fields in that region were not completed.

SMASH was allotted 57 nights of DECam observations (on 63 separate nights, with 12 of these being half nights) and 47 nights (or 75\%) of useful data were obtained. All allocated observing for the SMASH survey has now concluded. The median seeing in ugriz is (1".22, 1 "' 13,1 " 01,0 " 95,0 ".90), respectively, with a standard deviation of $\sim 0.25$ and the median airmass of all observations is 1.35 . Useful exposures were obtained for 197 fields (158 deep fields), but 27 fields from our original survey plan remain unobserved due to poor weather conditions (mainly in the Leading Arm region). Table 2 shows all SMASH survey observing time including all time and data from non-NOAO sources (which are all combined as part of the SMASH data set). More information about which nights were photometric are in the smash_observing_conditions.txt file (see Section 6.1.2 below).

\section{1. $0.9 \mathrm{~m}$ Observations}

The CTIO $0.9 \mathrm{~m}$ telescope and Tek $2 \mathrm{~K}$ CCD camera were used to collect observations of SDSS standards and SMASH fields in order to provide an independent calibration of a portion of the DECam data, particularly for fields observed under non-photometric conditions with the $4 \mathrm{~m}$ telescope. The bulk of these observations were taken using CTIO's SDSS ugriz filter set, while for three nights we used the borrowed DES PreCam griz filters ${ }^{36}$ for the sake of comparison.

The typical nightly observing plan in photometric weather was to observe several standard star fields from Smith et al. (2002) and from SDSS Stripe 82 and Stripe 10 (DR12; Alam et al. 2015) at the beginning and end of each night and every $\sim 2$ hours in between, and observe SMASH fields during the rest of the time. The Tek $2 \mathrm{~K}$ camera has a $13.5 \times 13.5$ field of view and so covered only the central portion of the SMASH fields. We did not offset the $0.9 \mathrm{~m}$ telescope to cover the full DECam field of view, and so obtained calibration information only for the central DECam chips. Typical exposure times for the standard fields were $300 \mathrm{~s}(u), 20 \mathrm{~s}(g), 5 \mathrm{~s}(r), 10 \mathrm{~s}(i)$, and $15 \mathrm{~s}(z)$, while for the SMASH fields we took sets of five undithered exposures with individual exposure times of $600 \mathrm{~s}$ $(u), 60 \mathrm{~s}(g), 60 \mathrm{~s}(r), 120 \mathrm{~s}(i)$, and $360 \mathrm{~s}(z)$. During nonphotometric $0.9 \mathrm{~m}$ nights, we only took images of SMASH fields and used short exposures of these fields taken on photometric nights to bootstrap the calibration of the nonphotometric exposures. Table 2 also summarizes the $0.9 \mathrm{~m}$ observing runs.

Calibration data taken at the telescope consist of daily dome flats in griz, twilight sky flats in ugriz, exposures for the creation of a shutter shading map, and exposures for the creation of a bad pixel mask. The shutter shading calibration data consist of $r$-band dome flats observed while repeatedly opening the shutter for one second and closing it during the exposure, intermingled with normal dome flats taken with the

\footnotetext{
36 The DES PreCam filters are a $4 \times 4$ inch filter set made to the same specification as the full-sized DECam filters.
} 
Table 2

SMASH DECam and $0.9 \mathrm{~m}$ Observing Runs

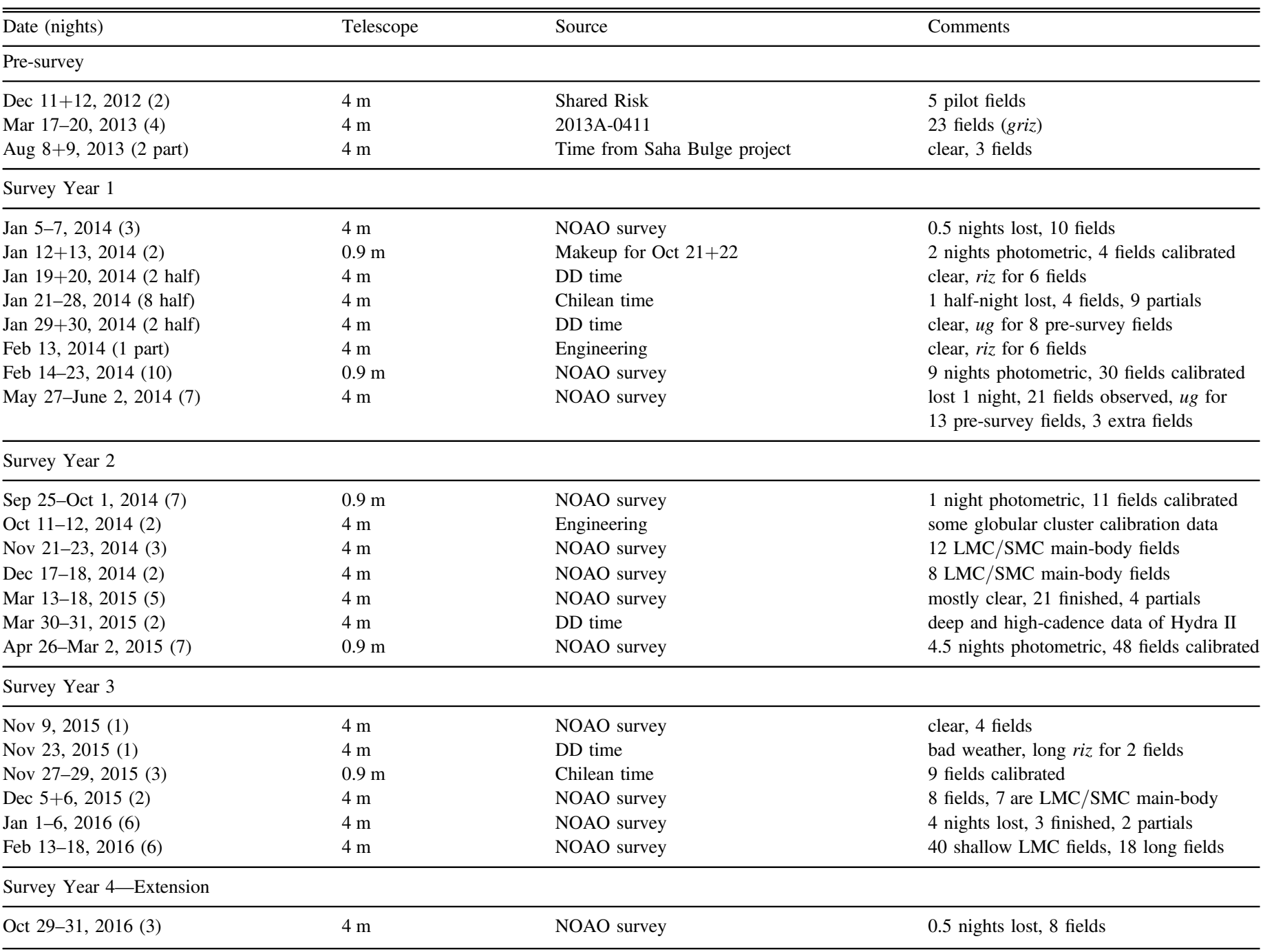

Note.

${ }^{a}$ Full up-to-date table is available in the obslog/smash_observingruns.txt file on the SMASHRED repository (https://github.com/dnidever/ SMASHRED).

same total exposure time as the shutter frames. The bad pixel mask data consisted of $1000.1 \mathrm{~s} r$-band dome flat exposures and a set of six $r$-band dome flats taken with levels equaling $75 \%$ of saturation.

\section{Image Processing}

This section describes the image processing from raw to flattened and "detrended" final images for both the DECam and $0.9 \mathrm{~m}$ data.

\subsection{Image Processing of the DECam Data}

The SMASH data reduction of the DECam data makes use of three separate software packages: (1) the NOAO Community Pipeline (CP; Valdes et al. 2014) ${ }^{37}$ for instrument signature removal (Section 4.1.1), (2) PHOTRED ${ }^{38}$ for point-spread-

\footnotetext{
37 http://www.noao.edu/noao/staff/fvaldes/CPDocPrelim/PL201_3.html; also see the NOAO Data Handbook: https://www.noao.edu/meetings/ decam/media/DECam_Data_Handbook.pdf.

38 https://github.com/dnidever/PHOTRED
}

function (PSF) photometry (Section 5.2), and (3) SMASHRED, a custom software written for PHOTRED pre- and postprocessing of the SMASH data (Section 5.1 and 6.3)).

\subsubsection{Community Pipeline Reductions}

The CP was developed by the Dark Energy Survey Data Management team but with subsequent important modifications by $\mathrm{F}$. Valdes at NOAO to produce reduced images for the community. The $\mathrm{CP}$ performs the following operations on the data:

1. Bias correction

2. Crosstalk correction

3. Saturation masking

4. Bad pixel masking

5. Linearity correction at both low and high count levels

6. Flat-field calibration

7. Fringe pattern subtraction, for $z$ and $Y$ bands

8. Bleed trail and edge bleed masking and interpolation 
9. Astrometric calibration of the image World Coordinate System (WCS) with 2MASS as the astrometric reference catalog

10. Single-exposure cosmic-ray masking by finding pixels that are significantly brighter than their neighbors

11. Photometric calibration using USNO-B1

12. Sky pattern removal; the "pupil ghost" and spatially varying background are subtracted

13. Illumination correction using a "dark sky illumination" image

14. Remapping to a tangent plane projection with constant pixel size

15. Transient masking with multiple exposures

16. Single-band coadding of remapped exposures with significant overlap

The CP is run by NOAO staff on all community DECam data. It generally takes a week after the completion of an observing run for the data to be processed and become available on the NOAO Science Archive. ${ }^{39}$ The CP produces instrumentally calibrated images ("InstCal"), remapped versions of InstCal ("Resampled"), and single-band coadded images ("Stacked"). For SMASH, we use the InstCal images, which come in three multi-extension (one per chip) and fpack ${ }^{40}$ compressed FITS files per exposure: flux ("image"), weight/ variance ("wtmap"), and quality mask ("qmask").

\subsection{Image Processing of the $0.9 \mathrm{~m}$ Data}

We used the NOAO/IRAF QUADRED package, custom IDL programs, and other software to process the images from the $0.9 \mathrm{~m}$ observations. The basic steps of this processing are:

1. Electronic crosstalk correction, using custom software to measure and correct for the electronic ghosting present in the images when read through multiple amplifiers.

2. Correction for electronic bias using the CCD's overscan region and bias frames.

3. Trimming of the images to the illuminated area.

4. Derivation of the exposure time-dependent illumination map caused by the opening and closing of the camera's iris shutter using dome flat observations designed for this purpose, and application of this shutter shading correction to the observed images.

5. Derivation of flat-field frames from twilight sky images and application to the object frames.

6. Derivation of a bad pixel mask from dome flat observations designed for this purpose, with bad pixel correction applied to the object frames.

7. Derivation of dark sky flats (ugri) and fringe frames (z) by stacking and filtering the deep sky observations taken throughout each observing run, followed by division by the dark sky flats (ugri) and subtraction of fringe features (z) for all object frames.

8. Use of the code library from http://astrometry.net (Lang et al. 2010) to populate the object image headers with WCS solutions.

\footnotetext{
39 https://www.portal-nvo.noao.edu

${ }^{40}$ https://heasarc.gsfc.nasa.gov/fitsio/fpack/
}

\section{Photometric Reduction}

This section describes how photometric catalogs were created from the reduced images of the DECam and $0.9 \mathrm{~m}$ data.

\subsection{DECam Pre-processing with SMASHRED}

The CP-reduced images are not in a format that is readable by DAOPHOT. Therefore, we run a SMASH pre-processing script (SMASHRED_PREP.PRO) on the CP images for each night before PHOTRED is run. This script performs the following steps:

1. Rename files in the old ("tu") naming convention to the new ("c4d") convention (the official NOAO archive file naming convention ${ }^{41}$ was changed in early 2015 ).

2. Move standard star exposures to the "standards/" directory since they are processed separately from the science data (see Section 6.1.1).

3. Uncompress the FITS files, set "bad" pixels to 65,000 , and write new FITS files for each chip image.

4. Sort the exposures into PHOTRED "fields" based on the pointing and exposure times (short and long exposures are processed separately). Rename the files using the PHOTRED file naming convention (FIELD\#-EXPNUM\#_CHIP\# .fits, e.g., F5-00507880_17.fits).

5. Download astrometric reference catalogs for each field and write separate reference catalog files for each chip FITS file.

6. Move files for each field into a separate directory (e.g., $\mathrm{F} 8 /, \mathrm{F} 9 /, \mathrm{F} 10 /, \mathrm{F} 11 /)$.

The CP mask files provide information on bad pixels, saturation, bleed trail, cosmic rays, and multi-exposure transients. There were some problems with the multi-epoch transient masking so we ignored that information in the mask. Any pixels that were affected by the other issues were set to a high value $(65,000)$ so that PHOTRED/DAOPHOT would see these pixels as "bad" and ignore them.

\subsection{DECam Nightly PHOTRED Reduction}

Accurate PSF fitting photometry was obtained using the automated PHOTRED pipeline first described in Nidever et al. (2011). PHOTRED performs WCS fitting, single-chip PSF photometry, as well as multi-exposure forced-PSF photometry using the DAOPHOT suite of programs (Stetson 1987, 1994). PHOTRED was run separately on each night. The short and long exposures of a field were run through PHOTRED separately (the former with a "sh" suffix added to their name), and multiband image stacking and forced photometry were only performed on the long exposures. This was mainly because of issues with bright, saturated stars when stacking short and long exposures, and the fact that the short exposures did not add much to the overall depth of the longer exposures. Note also that deep exposures of a field taken on different nights were processed separately and only combined during the calibration stage (see Section 6).

PHOTRED is based on methods and scripts developed by graduate students and postdocs in S. Majewski's "halo" group at the University of Virginia (UVa) in the late 1990s and early 2000s (in particular J. Ostheimer, M. Siegel, C. Palma,

\footnotetext{
$\overline{41}$ http://ast.noao.edu/sites/default/files/File_Naming_Conventions_ v12.pdf
} 
T. Sohn, and R. Beaton). PHOTRED fully automates these scripts (and some manual procedures) into a robust and easy-touse pipeline. Most of the PHOTRED software was written by D.L. Nidever in 2008 while he was a graduate student at UVa and has been continually updated and improved since then. PHOTRED consists of IDL ${ }^{42}$ driver programs wrapped around the DAOPHOT Fortran routines, but also includes some IRAF, Fortran, and Unix shell scripts.

PHOTRED currently has 13 "stages." Text-based lists are used for keeping track of inputs, outputs, and failures, and for shuffling files from one stage to the next. This overall design was taken partly from the SuperMACHO "photpipe" pipeline (Rest et al. 2005; Miknaitis et al. 2007). The global parameters and optional settings (see the github repository for the full list) as well as the stages to be run are specified in the photred. setup setup file. The majority of the stages work on a chipby-chip level (SPLIT-CALIB), and these chip-level catalogs are combined for the few final stages (COMBINE-SAVE). Although not all of them are used for SMASH, for completeness and future reference, all of the stages are described in detail below.

\subsubsection{RENAME}

The headers are checked for all the required keywords (gain, read noise, time stamp, filter, exposure time, $\alpha / \delta$, airmass). The exposures are grouped into "fields" based on values in the "object" keyword in the header and renamed with the PHOTRED naming convention (FIELD\#-EXPNUM\#_CHIP\#.fits). The PHOTRED short field names and full field names are saved in the fields file. This stage was skipped for SMASH since it is already performed by the SMASHRED_PREP. PRO pre-processing script.

\subsubsection{SPLIT}

If the FITS files are multi-extension files, then these are split into separate FITS files per chip. This stage was also skipped for SMASH.

\subsubsection{WCS}

The WCS for an image is created (or refined if it already exists in the header) by using an astrometric reference catalog and some information about the imager (pixel scale and orientation) and pointing (rough $\alpha / \delta$ of the center of the image, normally from the Telescope Control System). The software (WCSFIT.PRO) performs its own simple source detection, sky estimation, and aperture photometry of the image using routines from the IDL Astronomy User's Library. ${ }^{43}$ If a WCS does not already exist, then the reference catalog $\alpha / \delta$ values are transformed roughly to the $x / y$ Cartesian coordinates of the image by using the exposure and image information provided. The reference sources are then cross-matched with the image sources by cross-correlating downsampled "detection" map images of the two groups of sources. The peak in the crosscorrelation image is used to obtain an initial measurement of the $x / y$ offsets between the lists and the significance of the match. If a significant match is found, then nearest-neighbor matching is performed with a large matching radius and the

\footnotetext{
${ }^{42}$ The Interactive Data Language is a product of Exelis Visual Information Solutions, Inc., a subsidiary of Harris Corporation.

${ }^{43}$ http://idlastro.gsfc.nasa.gov
}

measured offsets. The matches are used to fit a four-parameter transformation matrix (essentially translation, rotation, and scale) and a second round of improved nearest-neighbor matching. The final matches are used to perform fitting of the four CD\#_\# and two CRVAL\# parameters of the WCS. The software does not create or modify existing higher-order distortion terms but uses them if they already exist in the header (e.g., for DECam, the existing PV\#_\#\# TPV distortion terms are used).

By default, the SMASHRED pre-processing used USNO-B $1^{44}$ (Monet et al. 2003) as the astrometric reference catalog, and sometimes 2MASS or UCAC4 (Zacharias et al. 2013). The rms of the residuals of the astrometric solutions with these catalogs gave values of $\sim 270$ mas. After the first Gaia data release (Gaia Collaboration et al. 2016), the WCS fitting software was rerun with Gaia as the astrometric reference and the resulting FITS header (with the improved WCS) saved in a separate text file (gaiawcs.head) for each image. The astrometric solutions were dramatically improved, with a resultant rms of only $\sim 20$ mas (see Figure 2).

\subsubsection{DAOPHOT}

This stage detects sources in the single-exposure images, constructs the PSF, and uses it to measure PSF photometry with ALLSTAR. There are several steps:

1. PSF FWHM Estimate: Since DAOPHOT requires an estimate of the PSF FWHM (full width at half maximum, or seeing), a custom IDL routine (IMFWHM.PRO) with independent algorithms is used to make this estimate. The routine detects peaks in the image $8 \sigma$ above the background (although this is lowered if none are detected) and keeps only those peaks having the maximum value within 10 pixels of the peak position and have two or more neighboring pixels that are brighter than 50\% of the peak's flux (to help weed out cosmic rays). The routine then finds the contour at half-maximum flux in a $21 \times 21$ skysubtracted subimage centered on the peak and uses it to measure an estimate of the FWHM $(2 \times$ the mean of the radius of the contour) and the ellipticity of the contour. In addition, the total flux in the subimage and a "round" factor (similar to the DAOPHOT version) using marginal sums are computed. These metrics are then used to produce a cleaner list of sources (FWHM $>0$, round $<1$, ellipticity $<1$, and flux $<0$ ) on which two-dimensional Gaussian fitting is performed and more reliable metrics are computed. The final list of sources is selected by cuts on the new metrics and the distributions of the semimajor axis, semiminor axis, and $\chi^{2}$ (selected sources must lie within the dominant clustering of these parameters for all sources). The final FWHM and ellipticity are then computed from these sources using robust averages with outlier rejection. This FWHM value is then used in the next step to set the DAOPHOT input options.

2. DAOPHOT option files: Both DAOPHOT and ALLSTAR require option files (.opt and .als.opt, respectively). Some of the most important default settings are shown in Table 3.

For some very crowded fields (e.g., Field35 and Field46), the default settings produced suboptimal results, and therefore, linear PSF spatial variations $(\mathrm{VA}=1)$ and

\footnotetext{
${ }^{44}$ http://tdc-www.harvard.edu/catalogs/ub1.html
} 

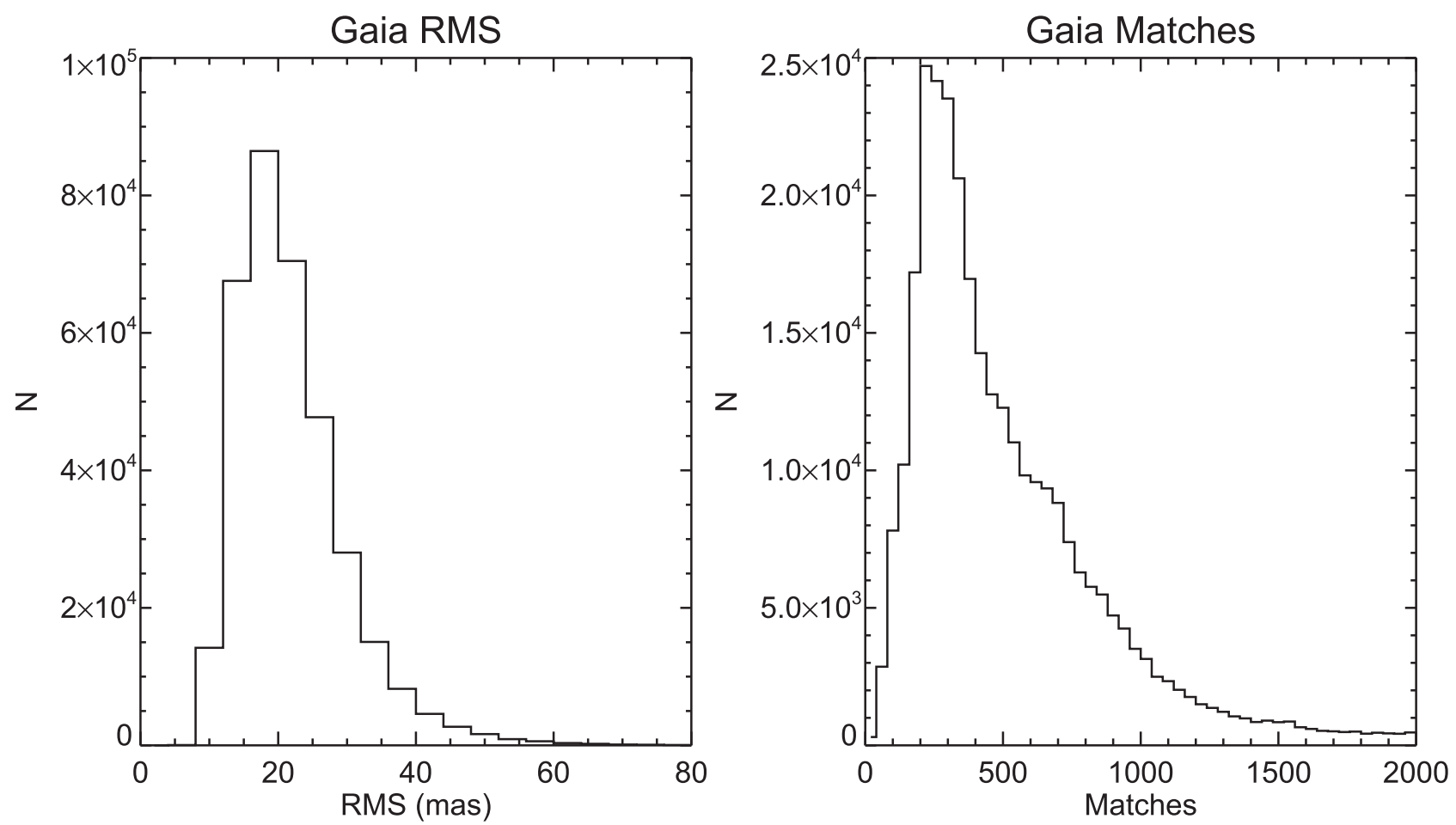

Figure 2. (Left) Distribution of rms values between the Gaia astrometric reference catalog and the SMASH DECam data for the final WCS (for the $\sim 350,000$ separate SMASH chip files). (Right) Distribution of matches per chip between the SMASH and Gaia data with a median of $\sim 500$ matches per chip.

Table 3

Default PHOTRED Options for DAOPHOT

\begin{tabular}{ll}
\hline \hline Option & Comment \\
\hline $\mathrm{TH}=3.5 \sigma$ & Detection threshold \\
$\mathrm{VA}=2$ & Quadratic spatial PSF variations \\
$\mathrm{FI}=1 \times$ FWHM & PSF fitting radius \\
$\mathrm{AN}=-6$ & Use lowest $\chi^{2}$ analytical PSF model \\
\hline
\end{tabular}

a smaller fitting radius $(\mathrm{FI}=0.75 \times \mathrm{FWHM})$ were used. The affected nights are 20141123, 20141217, 20151205 , and 20151206.

3. Common sources list: Early on in the development of PHOTRED, there were issues with constructing good PSFs for the deep (280 s), intermediate-band DDO51 observations for the MAPS survey (Nidever et al. 2011, 2013; which was the main motivation for writing PHOTRED). This was because there were a lot of point-like cosmic rays that overwhelmed the small number of real sources and made it difficult to create a good PSF source list just by culling via morphological parameters. To deal with this problem, PSF sources were required to be detected in multiple exposures (across all filters) to make sure they were real objects. In this step, a "common sources list" is constructed for each FITS image and later used as the starting point to select PSF stars. In the DECam data, the original issue is not as much of a problem because of the broad-band filters and because the cosmic rays in the LBNL detectors tend to be more linear and less point-like in shape. However, we continued to use the common sources option in the DAOPHOT stage for SMASH.
4. Detection: Sources are detected in the images with FIND, and aperture photometry is determined with PHOTOMETRY with an exponential progression of apertures from 3 to 40 pixels and sky radius parameters of 45 (inner) and 50 (outer) pixels.

5. Construct PSF: The PSF is constructed with an iterative procedure for culling out "suspect" sources. The initial list of 100 PSF sources is selected using PICK from the common source list (or the aperture photometry file if the common source option was not used), and a morphology cut is applied $(0.2 \leqslant$ sharp $\leqslant 1.0 ;$ using the sharp produced by FIND) to remove extended objects. The list is then iteratively cleaned of suspect sources. At each iteration, a new PSF is constructed with the PSF using the new list, and DAOPHOT prints out the "chi" value (root mean square (rms) residual of the stellar profile from the best-fitting analytic model) for each star and flags any outliers (? and $*$ for two and three times the average scatter, respectively). The flagged outliers and any sources with chi $>0.5$ are removed from the list, and the procedure is started over again until no more sources are rejected. In most cases, only one iteration is required.

After the list has converged, sources neighboring the PSF sources (using GROUP) are removed from the image (using SUBSTAR). A new PSF is constructed from this "neighbors subtracted" image and a similar iterative loop is used to remove PSF outlier sources. As before, most cases only require one iteration. The median number of PSF stars used per chip image is $\sim 80$.

The default PHOTRED setting is to allow DAOPHOT to pick the analytic function that produces the lowest chi value. The most commonly used function (77\% of the PSFs) is the four-parameter "Penny," which is the sum of a Gaussian and Lorentz function. The 

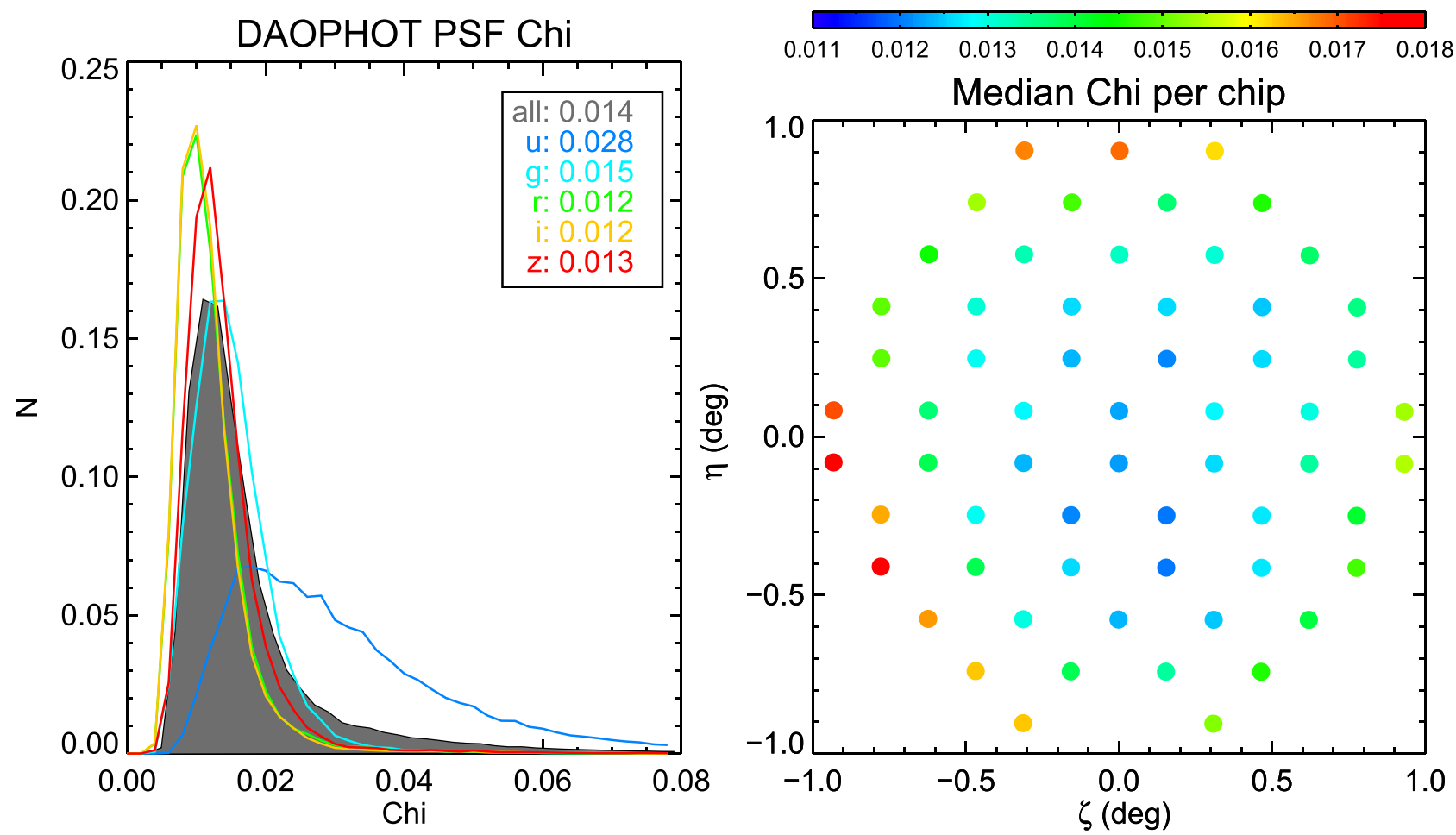

Figure 3. (Left) Distribution of DAOPHOT PSF "chi” values (relative rms of the analytic PSF residuals) for the $~ 350,000$ separate SMASH chip files broken down by band. The median values per band are given in the legend. (Right) Median chi value per chip (over all bands) as they appear in the focal plane.

second most common function (20\% of the PSFs) is a Moffat function with a power-law exponent of $\beta=3.5$.

6. Run ALLSTAR: ALLSTAR is run to perform simultaneous PSF fitting on all of the detected sources in the image using the constructed PSF. The default PHOTRED setting is to allow ALLSTAR to recentroid each source. ALLSTAR is also run on the "neighbors subtracted" images to obtain PSF photometry for the PSF stars that are later used to calculate an aperture correction. ALLSTAR outputs $x / y$ centroids, magnitudes with errors, sky values, as well as the chi and sharp (which describes how much broader the profile of the object appears compared to the profile of the PSF) morphology parameters (.als file).

One of the failure modes for a file in this stage is not to have enough PSF stars after the cleaning to constrain the solution. In these cases, the PSF spatial variation value (VA) is lowered in the option file by hand and DAOPHOT rerun. This solves the failures in the large majority of cases. For the small number of files where this also fails, we select PSF sources by visual inspection. The software will be modified to avoid these problems in the future by starting with a simple, constant analytic PSF and slowly adding more complexity if it is needed.

Figure 3 shows the histogram of DAOPHOT analytic PSF chi values broken down by band. The griz chi values are tightly peaked around $\sim 0.012$ (or $1.2 \%$ ), while the $u$-band values are a factor of $2 \times$ larger. The higher $u$-band values are because the $\mathrm{S} / \mathrm{N}$ of the PSF stars are on average lower, which gives rise to larger scatter in the residuals from photon noise. The right-hand panel of Figure 3 shows the median chi value per chip (across all exposures) as they appear on the sky, indicating that the analytic first approximations are slightly poorer for the chips on the periphery of the focal plane, possibly due to optical distortions or non-conformity of the detectors to the focal plane. Note that any systematic differences between the "true" PSF and the analytic first approximation go into DAOPHOT's PSF empirical look-up table of corrections, so the final rms of the PSF residuals will actually be smaller than the chi values. Figure 4 shows the diagnostic thumbnails of the mediancombined relative flux residuals from PSF-subtracted images of many bright stars. The PSF relative flux error is of the order of $\sim 0.3 \%$ with very little systematic structure left in the medianed residual image, indicating that the PSFs are of high quality.

\subsection{5. $\mathrm{MATCH}$}

The sources in the ALLSTAR photometry catalogs from the DAOPHOT stage are cross-matched and combined with the files for each chip being handled separately (i.e., all of the chip 1 files are cross-matched together, and all of the chip 2 files are cross-matched together, etc.). Astrometric transformations between the frames (using the $x / y$ Cartesian coordinates) and a reference frame (which is chosen based on the longest exposure time frame in the filtref band) are computed (in a similar manner to what DAOMATCH achieves). The WCSs in the FITS headers are used to calculate an initial estimate of the transformations. If this fails, then a more general matching routine that uses a cross-correlation technique of a downsampled "detection" map image between the two source lists (as described above in Section 5.2.3) is run. Once all of the transformations are in hand, DAOMASTER is used to iteratively improve the transformations (written to the .mch file) and cross-match, and finally, to combine all of the photometry (per chip) into one merged file (the . raw file). 


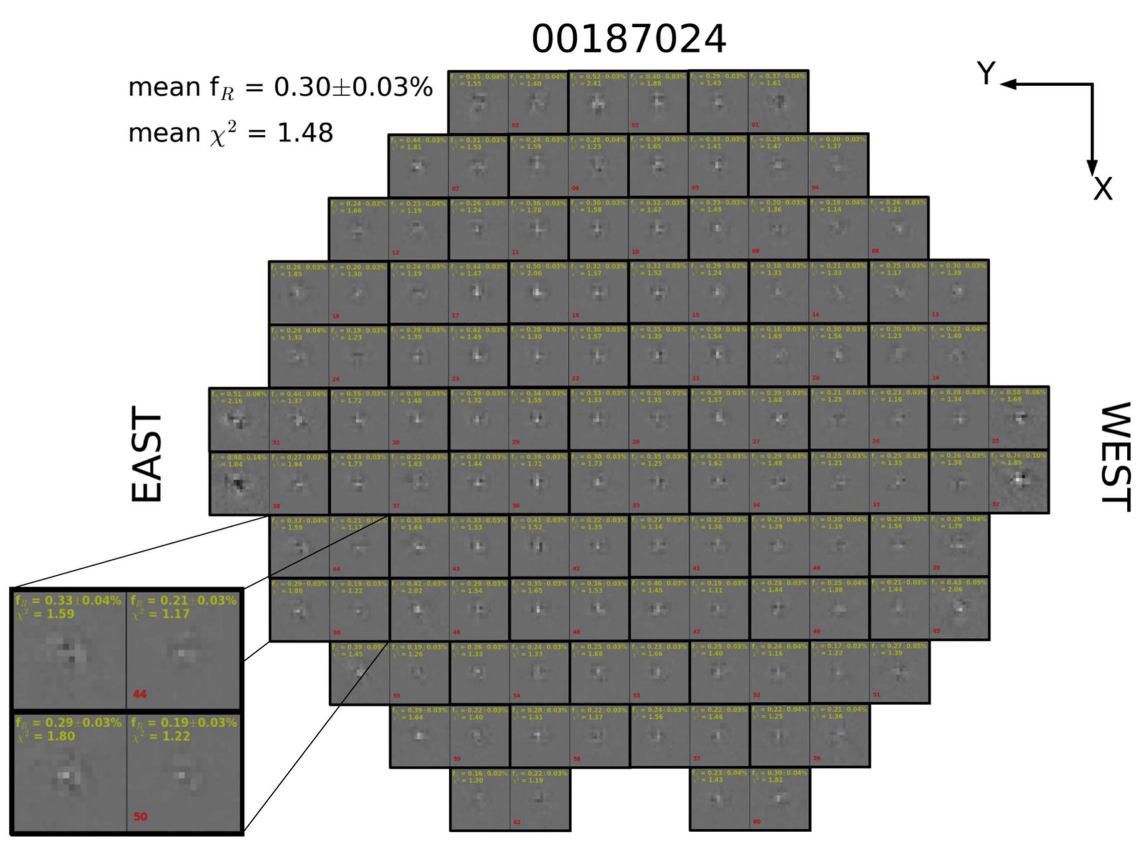

Figure 4. PSF quality assurance figure for the full focal plane of a single exposure (EXPNUM $=00187024)$ and a blow-up of two chips in the lower left. Each square image shows the relative residuals in the PSF-subtracted image (relative to total flux in the PSF model) medianed across $\sim 30$ high S/N stars (not necessarily PSF stars) per half-chip. Each horizontal rectangle represents one chip of an exposure, and the two squares in the rectangle show the relative residuals for one half of the chip. The relative absolute residuals and the uncertainties (using propagation of errors from the noise in each image) as well as the $\chi^{2}$ for each half-chip are shown in the top of each square in yellow. The range of the grayscale is $\pm 0.2 \%$ and the chip number (CCDNUM) is shown in red. Average values for the entire exposure are in the upper-left corner.

\subsubsection{ALLFRAME}

PSF photometry can be improved by using a stacked image for source detection and then holding the position of the sources fixed while extracting PSF photometry from each image. The improvement of this "forced" photometry over regular PSF photometry performed separately from each image comes from the reduced number of free parameters (i.e., the positions). PHOTRED makes use of the DAOPHOT ALLFRAME (Stetson 1994) program to perform the forced photometry. The ALLFRAME stage processes each group of chips separately (e.g., chip 01 files separately from chip 02 files, etc.).

This stage performs several separate tasks:

1. Construct multiband coadd: A weighted-average stack is created of all images. First, the relative flux scaling, sky level, and weights are computed for all of the images. The weights are essentially the $\mathrm{S} / \mathrm{N}$ and are based on sources detected in all of the images (if no sources are detected in all of the images, then a bootstrap approach is used to tie the images to one another). Second, images are transformed to a common reference frame. The original code only applied $x / y$ translations to the images. However, this was insufficient for larger dithers where the higher-order optical distortions become important, and the software was rewritten to fully resample the images onto the final reference frame. The type of transformation used can be found in the ALFTILETYPE column ("ORIG" or "WCS") of the final chips catalog/ table. Finally, the images are average-combined using the IRAF routine IMCOMBINE with bad pixel masking and outlier rejection (sigma clipping). The detector gain recorded in the image header is maintained because the images are scaled to the reference exposure. However, new read noise and sky values ${ }^{45}$ for the combined image (_comb.fits) are computed using the weights, scalings, and sky values. It is challenging to preserve the fidelity of bright stars when combining deep and shallow exposures. This is one reason why it was decided that short and long SMASH exposures will be processed separately in PHOTRED.

2. PSF construction: The PSF of the combined image is constructed using the same routine as in the DAOPHOT stage.

3. Iterative source detection: Source detection is performed iteratively in two steps. (1) Detect new sources in the working image (PSF subtracted after the first iteration) with Source Extractor (SExtractor; which works well for detecting faint sources; Bertin \& Arnouts 1996) and incorporate these new detections into the master source list. (2) ALLSTAR is run on the original image (with the previously found PSF) using the current master source list, and all sources that converge are subtracted. This two-step process is repeated for the desired number of iterations (finditer option in photred.setup). The ALLSTAR output from the last iteration is used as the final master source list (_comb_allf.als). The default detection settings for SExtractor are as follows: use a convolution filter, $>1 \sigma$ detection threshold, and a minimum area of 2 pixels per source. For SMASH, only two iterations were used since we found that further iterations produced mainly spurious new detections.

\footnotetext{
$\overline{45}$ Sky is needed in the combined images because DAOPHOT uses it as part of its internal noise model.
} 
4. Run ALLFRAME: ALLFRAME is run on all of the images using their respective PSFs and the master source list constructed in the previous step. ALLFRAME uses the coordinate transformations between images from the . mch file (in the MATCH stage), but computes its own small, high-order geometric adjustments (we use the option of 20 terms or cubic in $x$ and $y$ ) to these during the fitting process (it slowly adds in the higher orders to keep the solutions constrained). We allow a maximum of 50 iterations in ALLFRAME after which it outputs catalogs (.alf) with $x / y$ coordinates (in that image's reference frame), photometry with errors, and the chi and sharp morphology parameters.

After ALLFRAME has finished, the results for the individual images are combined and the SExtractor morphology parameters are added to the final catalog (.mag file).

It is possible to skip the ALLFRAME stage for certain fields by specifying them in the alfexclude option of the photred.setup file. This option was used for the short SMASH exposures.

\subsubsection{APCOR}

The DAOPHOT program DAOGROW (Stetson 1990) is used to produce growth curves for each band and night separately. These are used to produce "total" photometry (including the broad wings) for the bright PSF stars for each chip. These values are then compared to the PSF photometry values for the PSF stars (from neighbor-subtracted images) produced in the DAOPHOT stage to compute an average aperture correction for each chip. These values are all stored in the apcor. lst file and used later in the CALIB stage.

\subsubsection{ASTROM}

The WCS in the FITS header is used to add the $\alpha$ and $\delta$ coordinates for each object to the catalog.

\subsection{9. $C A L I B$}

The photometry is calibrated using the transformation equations given in the transformation file specified in photred.setup (e.g., n1.trans). The equations in the file can pertain to various levels of specificity: (1) only the band is specified, (2) the band and chip are specified, or (3) the band, chip, and night are specified. The terms in the transformation file are zero point, extinction, color, extinction $\times$ color, and color $\times$ color, along with their uncertainties. Besides these corrections, the photometry is also corrected for the exposure time and the aperture correction (for that chip).

Because the calibrated color is used for the color term, the software uses an iterative method to calibrate the photometry (using an initial color of zero). A weighted-average value from all exposures is used for the magnitude in the other band to construct the color (if multiple exposures in that band were taken), but not for the band being calibrated (the value for that exposure is used). Also, a color of zero is used for objects for which a good color cannot be constructed. The loop to derive the true source color continues until convergence (all magnitude differences are below the $0.1 \mathrm{mmag}$ level or 50 iterations, whichever is first).

Calibrated photometry for each exposure (e.g., G2, Z4) is given in the output file, and optionally, the average magnitudes per band (e.g., GMAG, ZMAG) and the instrumental magnitudes for each exposure (e.g., I_G2, I_Z4). Because a global calibration strategy was adopted for SMASH, all of the values in the transformation file were set to zero so that the photometry was only corrected for the exposure time and aperture corrections.

\subsubsection{COMBINE}

The individual chip catalogs are combined to create one catalog for the entire field. Sources detected in multiple chips (from dithered exposures) are combined and their photometry combined. The default matchup radius is $0 . " 5$ ( $\sim 2$ pixels).

\subsubsection{DEREDDEN}

Schlegel et al. (1998; SFD) $E(B-V)$ extinction values for each source are added as a separate column in the final, combined catalog. Extinction $(A[X])$ and reddening $(E[X-Y])$ values for the bands and colors specified in the photred. setup setup file (using $A[X] / E[B-V]$ values from the given extinction file) are also added to the catalog. The ugriz reddening coefficients from Schlafly \& Finkbeiner (2011) were used for SMASH.

\subsubsection{2. $S A V E$}

The final ASCII catalog is renamed to the name of the field (e.g., F5 is renamed to Field62) and a copy is created in the IDL "save" and FITS binary table formats. In addition, a useful summary file is produced with information on each exposure and chip for that field.

\subsubsection{HTML}

This stage creates static HTML pages to help with quality assurance of the PHOTRED results. Quality assurance metrics are computed and plots created for the pages. This stage was skipped for SMASH since custom quality assurance routines were written.

\section{3. $0.9 \mathrm{~m}$ Photometry}

We performed photometry on the $0.9 \mathrm{~m}$ observations of the SDSS standards and SMASH target fields with a pipeline based on the DAOPHOT software suite (by K.O., separate from PHOTRED/STDRED). In short, we used DAOPHOT to measure aperture-based photometry of the standard star frames, with a smallest aperture of $6^{\prime \prime}$ diameter $(\sim 15$ pixels $)$ and a largest aperture of $15^{\prime \prime}$ diameter ( $\sim 38$ pixels). We used DAOGROW to measure the growth curve based on the aperture measurements and to extrapolate the total instrumental magnitudes of the standard stars. These total instrumental magnitudes were then used as input to compare with the standard magnitudes in our derivation of the photometric transformation equations from the standard star observations, described in full below. We also measured the PSF photometry of the SMASH target fields and the standards using DAOPHOT and ALLSTAR. We derived PSFs from the images using as many as 200 point sources per image, using an iterative method to remove neighbors from the PSF stars and to improve the PSF estimation. We applied aperture corrections to the PSF photometry by comparing the PSF measurements with the total instrumental magnitudes from DAOGROW and fitting for the residuals with second-order polynomial functions in $x$ 
and $y$. These aperture-corrected instrumental PSF magnitudes were used as the input when we derived standard magnitudes for the SMASH fields, described below. We also measured instrumental PSF magnitudes for the standard fields to make sure that the PSF photometry procedure did not introduce any systematic errors, also described below.

\section{Calibration}

The southern sky that SMASH is observing has not been well covered with ugriz CCD imaging, which means that it is not possible to calibrate our photometry with existing catalogs (in the same area of the sky) as can be done in the north by using SDSS and Pan-STARRS1 (Chambers et al. 2016) data. Therefore, we must use the traditional techniques of calibrating our data with observations of standard star fields (on photometric nights) and extra calibration exposures (for nonphotometric nights). Our overall calibration philosophy is as follows:

1. For photometric DECam nights, calibrate the data using photometric transformation equations derived using DECam exposures of SDSS equatorial fields.

2. For non-photometric DECam data, set the zero points using photometric DECam data of the same field or overlapping neighboring fields (only available in the LMC/SMC main bodies).

3. For SMASH fields with no photometric DECam data, the calibrated $0.9 \mathrm{~m}$ photometry for the central region is used to set the zero points.

It is important to note that SMASH is on a quasi-SDSS photometric system. The data were obtained through the DECam passbands and calibrated onto the SDSS system using zero points and linear color terms. However, the data were not corrected for nonlinear effects that arise because of the differences in the SDSS and DECam passbands, which are significant for the $r$-band and $u$-band. Therefore, the SMASH colors of stars can deviate from how they would appear in SDSS in certain bands for very red or blue stars. Caution should be used when comparing the data to other catalogs or model isochrones in these situations.

\subsection{Standard Star Calibration of DECam Data}

On every DECam night, "standard star" observations in the ugriz bands were taken every couple of hours of the SDSS equatorial fields at both high and low airmass. The equatorial fields lie in Stripe 82 and Stripe 10, and are the same as those chosen by DES to regularly sample the full range in RA. We downloaded "reference" catalogs via CasJobs ${ }^{46}$ for the equatorial fields from SDSS DR12 (Alam et al. 2015). These observations generally provided several thousands of "standard star" measurements per exposure and good color coverage.

\subsubsection{Processing of Standard Star Data with STDRED}

To reduce the DECam standard star exposures, we use the STDRED pipeline, which is a sister package to PHOTRED and works in a similar manner. The same SMASHRED_PREP. PRO pre-processing script is used to uncompress, mask, and split the $\mathrm{CP}$-reduced images and download the astrometric reference

\footnotetext{
46 http://casjobs.sdss.org
}

catalogs per field. The main STDRED steps that are used by SMASH are:

1. WCS: fits the chip WCS using the astrometric reference catalog.

2. APERPHOT: detects sources and performs aperture photometry.

3. DAOGROW: calculates aperture corrections via curves of growth and applies them to the aperture photometry.

4. ASTROM: adds $\alpha / \delta$ coordinates to the photometric catalog.

5. MATCHCAT: cross-matches the observed catalog with the reference catalog and for matches, outputs merged information from both catalogs.

6. COMBINECAT: combines all of the matched photometry for a given filter.

7. FITDATA: fits photometric transformation equations (with zero-point, color, and extinction terms) for each filter using all of the data. However, new custom software was developed to derive these equations for SMASH (see the next section).

The standard star exposures from each DECam run are processed with STDRED in their own directory.

\subsubsection{Derivation of DECam Photometric Transformation Equations from Standard Star Data}

To produce the highest-quality and most uniform calibration, we decided to write new custom software to determine the DECam photometric transformation equations using all of the standard star data together. Transformation equations of the following form were used:

$$
\begin{aligned}
& m_{\mathrm{obs}}=m_{\mathrm{cal}}+\text { ZPTERM }+ \text { COLTERM } \times\left(\text { color }_{\mathrm{cal}}\right) \\
& \quad+\text { AMTERM } \times X,
\end{aligned}
$$

where ZPTERM is the zero-point term, COLTERM is the color term, color $_{\text {cal }}$ is the calibrated color (which includes the band being calibrated), AMTERM is the extinction term, and $X$ is the airmass.

The new software (SOLVE_TRANSPHOT.PRO) has several options for what variables to fit or hold fixed (zero-point, color, extinction, and color $\times$ extinction terms) and over what dimensions (e.g., night and chip) to average or "bin" values. At first, all variables (zero-point, color, and extinction terms) were fit separately for each night and chip combinations to see how much the terms vary and over what dimensions.

Color. We found that the color terms vary from chip to chip (at the $\sim 0.01 \mathrm{mag}$ level as also noted on the DECam Calibration Web pages ${ }^{47}$; see the left-hand panel of Figure 5), but they appear to be temporally stable (see the bottom panel of Figure 5). Therefore, we fit the (linear) color terms for each chip separately by taking a robust average over all photometric nights.

No evidence for systematics was found in the color residuals of $g / i / z$, indicating there was no need for higher-order color terms. For the $u$-band, there are systematics in the residuals (consistent across all fields) that would require higher-order terms to fit. This is largely because of the different throughput curves for the SDSS and DECam filters. We decided not to add higher-order terms as these could adversely affect very blue or

\footnotetext{
47 http://www.ctio.noao.edu/noao/node/3176
} 

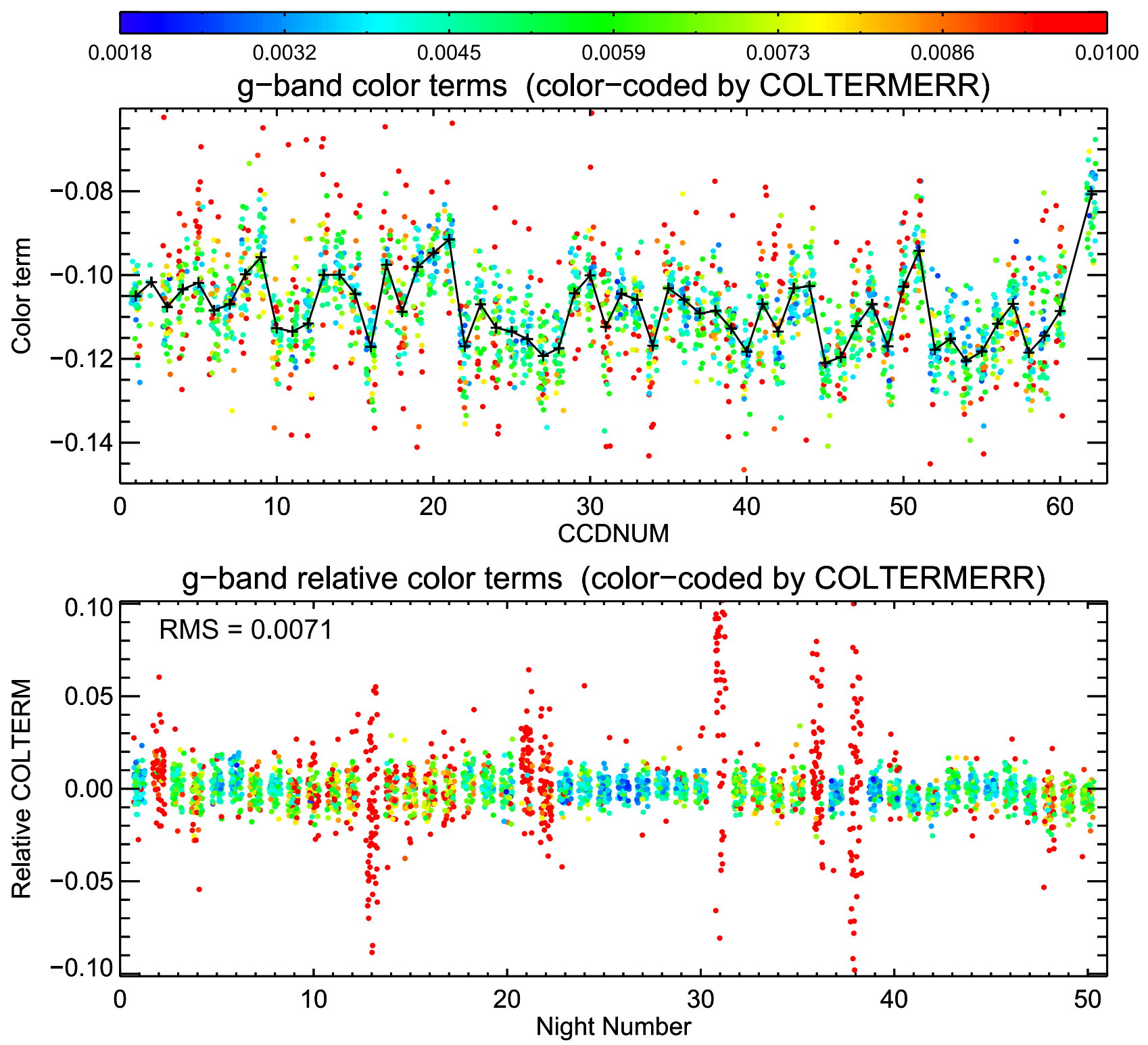

Figure 5. $g$-band color terms of the DECam photometric transformation equations. (Top) Dependence of the color terms on chip number. (Bottom) Temporal dependence of the "relative" color terms with the median color term of each chip subtracted ("night number" is a running counter of SMASH nights). The nights with large scatter are non-photometric nights.

red objects (where the solution is not well-constrained). However, to determine a uniform and reliable zero point, we decided to fit the shape in the residuals and remove this pattern from the observed data at the very beginning of the procedure. In addition, we restricted the color range to $1.0<u-g<2.5$. After this correction and color restriction are applied, the residuals are flat. We similarly restrict the color range for the $r$ band $(g-r<1.2)$ because the correlation between the SDSS and DECam $r$-band magnitudes becomes nonlinear for redder stars due to the difference in the throughput curves.

Extinction. An appreciable number of nights had a small range in airmass for the standard star observations that produced unreliable extinction term measurements. Therefore, for these nights, we calculated a weighted (by uncertainty and time difference) average of the nightly extinction terms for the four closest neighboring good nights. Similarly, for nights with larger airmass ranges, we improve the accuracy by refitting the extinction term using the individual data points from the four closest good neighboring nights (but these must be within 30 days). Finally, we found that there was no appreciable color $\times$ extinction dependence, and therefore, these terms were not included in the fits.

Zero Point. We tried separating the zero -points into nightly zero points and relative chip-to-chip (for each band but constant with time) zero-point offsets, considering that although the zero point can change nightly, due to transparency and extinction variations, the zero points of one chip to another (in a given band) should remain the same. We found, however, that the scatter in the relative chip-dependent zero points over the many nights was somewhat higher than was anticipated (but still small at $\sim 0.01$ mag), and we obtained better results by fitting a zero point for each night and chip combination. Therefore, we adopted the latter strategy and "abandoned" the relative zero points (although they are computed and saved in the final output file). 
Table 4

SMASH Median Photometric Transformation Equations

\begin{tabular}{llrrr}
\hline \hline Band & Color & Zero-point Term & Color Term & Extinction Term \\
\hline$u$ & $u-g$ & $1.54326 \pm 0.0069$ & $0.0142 \pm 0.0041$ & $0.3985 \pm 0.00240$ \\
$g$ & $g-r$ & $-0.3348 \pm 0.0019$ & $-0.1085 \pm 0.0010$ & $0.1747 \pm 0.00076$ \\
$r$ & $g-r$ & $-0.4615 \pm 0.0018$ & $-0.0798 \pm 0.0011$ & $0.0850 \pm 0.00098$ \\
$i$ & $i-z$ & $-0.3471 \pm 0.0016$ & $-0.2967 \pm 0.0012$ & $0.0502 \pm 0.00058$ \\
$z$ & $i-z$ & $-0.0483 \pm 0.0023$ & $-0.0666 \pm 0.0016$ & $0.0641 \pm 0.00075$ \\
\hline
\end{tabular}

Photometric nights are determined by seeing if the observers noticed any sign of clouds, looking for cloud cover in the CTIO RASICAM all-sky infrared videos, ${ }^{48}$ and finally, by looking at the scatter in the standard star residuals. The full list of nights for which STDRED was run and the photometric status are given in smash_observing_conditions.txt (in SMASHRED/obslog/).

The $\sim 3100$ variables (zero points for $\sim 50$ nights $\times 60$ chips, color terms for 60 chips, extinction terms for $\sim 50$ nights) were not fit to the data simultaneously but were found through an iterative fitting process:

1. Fit all terms separately for all night and chip combinations.

2. Compute the mean color term per chip using only photometric data.

3. Fix color terms and refit zero-point and extinction terms.

4. Average extinction terms. For photometric nights with poor solutions or low airmass ranges, a weighted average of the extinction terms of the nearest four neighboring photometric nights is computed. For the rest of the photometric nights, a new extinction term is computed using data included from the four nearest neighboring nights. The extinction term is set to zero for nonphotometric nights.

5. Fix color and extinction terms, and refit zero-point terms.

Although solutions are found for all nights, only the transformation equations for photometric nights are used to calibrate the data.

The final photometric transformation equations are written to file (smashred_transphot_eqns.fits available in SMASHRED/data/) with zero-point, color, and extinction terms (with uncertainties and averaging information) for each night and chip combination, as well as separate tables with information unique to each chip (e.g., color term) and information unique to each night (e.g., extinction term). The formal uncertainties on the terms are $\sim 0.002, \sim 0.0015$, and $\sim 0.0007$ for the zero point, color and extinction, respectively. For an average color and airmass, this amounts to a formal uncertainty in the photometry of $\sim 0.002 \mathrm{mag}(0.009 \mathrm{mag}$ for $u$ ). Table 4 gives the median values and uncertainties per band, while example residuals versus chip, airmass, and color for a single night are shown in Figure 6.

The nights of the UT 2014 January 5-7 observing run were clear and photometric but no SDSS standard star observations were taken. Therefore, the regular procedures could not be used to determine the transformation equations for these nights. Subsequently, some of the fields from this run could be calibrated because they were reobserved on other photometric

\footnotetext{
48 http://www.ctio.noao.edu/noao/node/2253
}

nights (with standard star data) or $0.9 \mathrm{~m}$ calibration data were obtained. The photometric transformation equations were then determined ("backed-out") by using these calibrated fields and using the previously derived chip-dependent color terms. The smashred_transphot_eqns.fits file was then updated with these values, and the data for those nights could be calibrated in the regular manner.

\subsection{Calibration of $0.9 \mathrm{~m}$ Data}

Using the aperture-corrected $0.9 \mathrm{~m}$ photometry, we explored fits to equations of the form:

$$
\begin{aligned}
u_{\mathrm{obs}} & =A_{1} u+A_{2} X+A_{3} x+A_{4} y+A_{5} t+A_{6}(u-g)+A_{7} \\
g_{\mathrm{obs}} & =B_{1} g+B_{2} X+B_{3} x+B_{4} y+B_{5} t+B_{6}(g-r)+B_{7} \\
r_{\mathrm{obs}} & =C_{1} r+C_{2} X+C_{3} x+C_{4} y+C_{5} t+C_{6}(g-r)+C_{7} \\
i_{\mathrm{obs}} & =D_{1} i+D_{2} X+D_{3} x+D_{4} y+D_{5} t+D_{6}(r-i)+D_{7} \\
z_{\mathrm{obs}} & =E_{1} z+E_{2} X+E_{3} x+E_{4} y+E_{5} t+E_{6}(i-z)+E_{7},
\end{aligned}
$$

where $u_{\mathrm{obs}} g_{\mathrm{obs}} r_{\mathrm{obs}} i_{\mathrm{obs}} z_{\mathrm{obs}}$ are the instrumental magnitudes, ugriz are the standard SDSS magnitudes drawn from Smith et al. (2002) and from SDSS DR12 (Alam et al. 2015), $X$ is the airmass, $x$ and $y$ are the pixel positions on the detector, and $t$ is the time of observation during the night.

We fit this set of equations first to the data taken on the almost entirely photometric run from 2014 February 14-23. Table 5 shows the best-fit coefficients. Although we fit the transformation equations independently on each of the 10 nights, we show only the average coefficients and their standard deviations in the table, as values were in all cases consistent across the nights. From our fits, we found no evidence for strong pixel position-dependent or time-dependent terms. We did, however, find evidence for a small magnitudedependent scale factor of $0.1 \%-0.5 \%$, which may point to a small nonlinearity with the Tek2K CCD.

We next explored fits to the equations for the observing runs on 2014 September 25-October 2, 2015 April 26-May 3, and 2015 November 27-29. These runs were complicated by variable weather conditions, work on the camera electronics that changed the gain setting of the CCD, and by a temporary change from the CTIO SDSS griz filter set to the PreCam griz filter set that more closely matches the filter set used by DECam. For these observations, we fit the SDSS and PreCam sets separately. For each filter set, we first used all of the nights observed with that set to measure the color-term coefficients. To do this, we allowed the zero point for each frame to be fit independently, which removes all other variables from consideration other than the color term; this allowed us to use standards taken on non-photometric nights to constrain the color term. We then fixed the color-term coefficients to these fitted values, and for the photometric nights, we fit for the remaining coefficients on a per night basis. For these fits, 

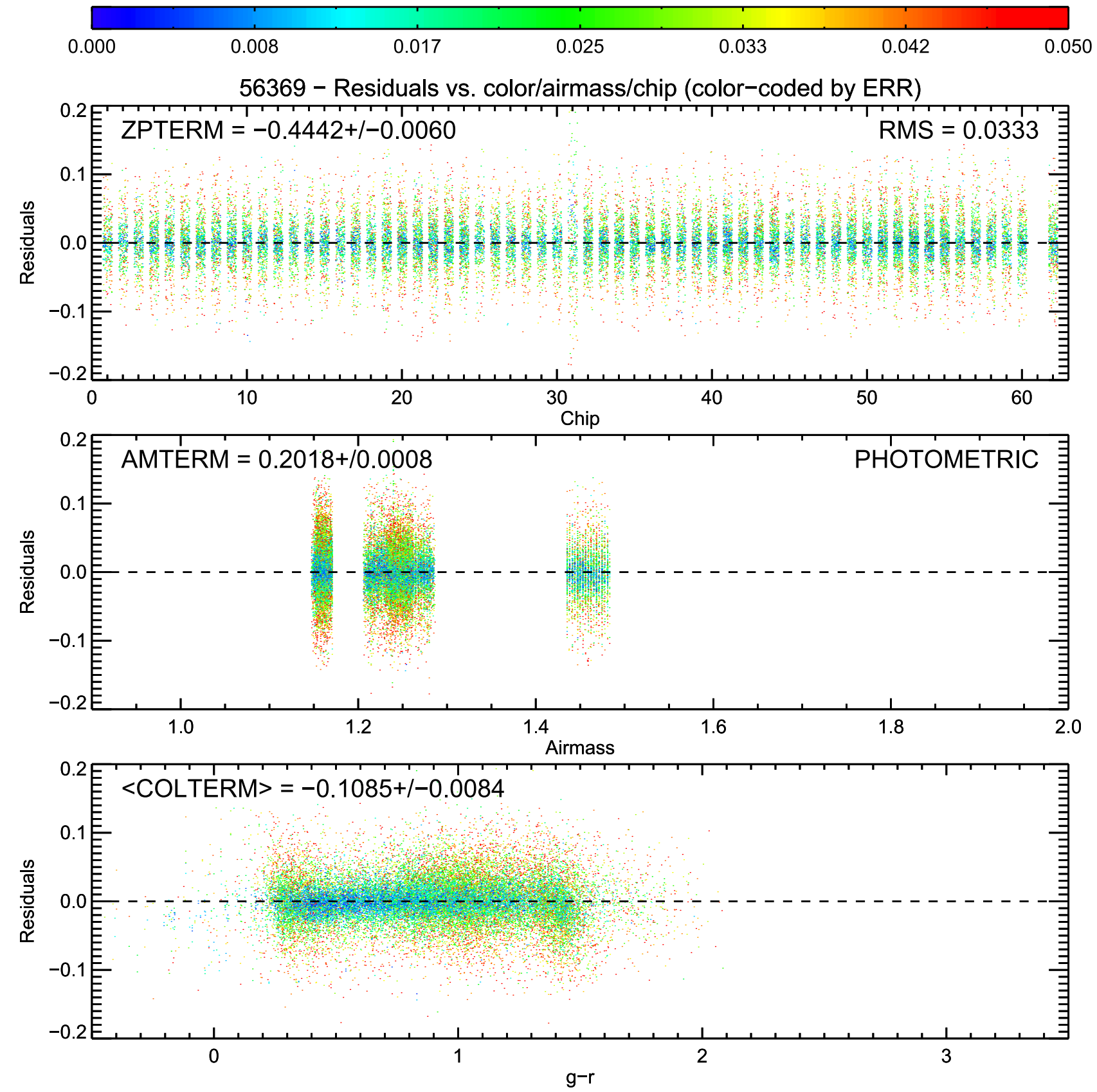

Figure 6. $g$-band residuals (SDSS reference magnitude - derived magnitude using the transformation equations) after fitting the photometric transformation equations to standard star observations for a typical photometric night. The observations are color-coded by their photometric error. (Top) Residuals vs. chip number. (Middle) Residuals vs. airmass. (Bottom) Residuals vs. $g-r$ color. The derived terms of the photometric transformation equations and their uncertainties are given in the upper-left-hand corner of their respective panel.

we found no evidence for pixel position-dependent, timedependent terms, or in contrast to the 2014 February observations, a magnitude-dependent scale term. Table 5 shows the fitted coefficients for the SDSS filters, where the larger standard deviation in the tabulated zero point reflects the large span of time over which the observations were taken. Figure 7 shows the photometric residuals for the standard star fields. Table 6 shows the color terms for the DES PreCam filter set for which we did not derive full transformation equations because of weather that was not completely photometric. We found some significant differences between the DES PreCam and SDSS color terms, particularly for the $r, i$, and $z$ filters, as is to be expected from the differences in the bandpasses. The color terms are not, however, identical to those measured with DECam, which we ascribe to differences in the PreCam bandpasses compared to DECam, as well as to differences in the telescope and detector response functions. In the end, we did not use the PreCam data for calibration, but present the results to verify that using a filter set that is a closer match to those used with DECam reveals systematic differences with the SDSS filters.

\subsection{Calibration Software}

New software was developed to perform calibration of SMASH fields across multiple nights and using a variety of zero-point calibration methods. The software also takes 
Table 5

$0.9 \mathrm{~m}$ Photometric Transformation Equations (SDSS Filter Set)

\begin{tabular}{|c|c|c|c|c|c|c|c|}
\hline Band & $\begin{array}{l}\text { Scale Factor } \\
(A B C D E)_{1}\end{array}$ & $\begin{array}{l}\text { Extinction } \\
(A B C D E)_{2}\end{array}$ & $\begin{array}{c}x \text { Factor } \\
(A B C D E)_{3}\end{array}$ & $\begin{array}{c}y \text { Factor } \\
(A B C D E)_{4}\end{array}$ & $\begin{array}{l}\text { Time Factor } \\
(A B C D E)_{5}\end{array}$ & $\begin{array}{l}\text { Color Term } \\
(A B C D E)_{6}\end{array}$ & $\begin{array}{l}\text { Zero Point } \\
(A B C D E)_{7}\end{array}$ \\
\hline \multicolumn{8}{|c|}{2014 Feb $14-23$} \\
\hline$u$ & $1.001 \pm 0.002$ & $0.51 \pm 0.02$ & $\equiv 0.0$ & $\equiv 0.0$ & $\equiv 0.0$ & $-0.034 \pm 0.004$ & $4.59 \pm 0.04$ \\
\hline$r$ & $0.995 \pm 0.001$ & $0.11 \pm 0.01$ & 0.0 & 0.0 & 0.0 & $-0.022 \pm 0.007$ & $2.67 \pm 0.02$ \\
\hline$i$ & $0.995 \pm 0.002$ & $0.06 \pm 0.01$ & 0.0 & 0.0 & 0.0 & $-0.017 \pm 0.014$ & $3.13 \pm 0.03$ \\
\hline$z$ & $0.998 \pm 0.001$ & $0.07 \pm 0.02$ & 0.0 & 0.0 & 0.0 & $0.040 \pm 0.011$ & $3.95 \pm 0.02$ \\
\hline$g$ & 1.0 & $0.18 \pm 0.01$ & 0.0 & 0.0 & 0.0 & $0.005 \pm 0.011$ & $2.51 \pm 0.31$ \\
\hline$r$ & 1.0 & $0.10 \pm 0.01$ & 0.0 & 0.0 & 0.0 & $-0.028 \pm 0.007$ & $2.49 \pm 0.21$ \\
\hline$i$ & 1.0 & $0.06 \pm 0.01$ & 0.0 & 0.0 & 0.0 & $-0.026 \pm 0.014$ & $2.91 \pm 0.12$ \\
\hline$z$ & 1.0 & $0.06 \pm 0.01$ & 0.0 & 0.0 & 0.0 & $0.022 \pm 0.011$ & $3.72 \pm 0.05$ \\
\hline
\end{tabular}
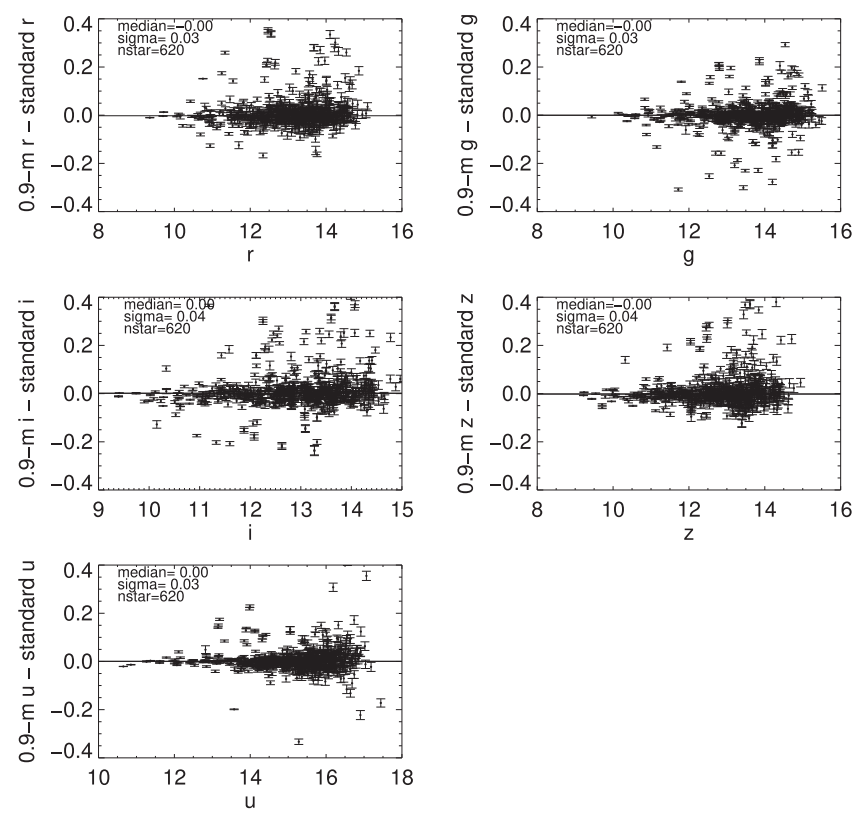

Figure 7. Residuals of the $0.9 \mathrm{~m}$ photometry relative to the standard star data vs. magnitude for the ugriz bands. Statistics for the residuals are in the upperleft-hand corner indicating rms values of 0.03-0.04 mag.

Table 6

$0.9 \mathrm{~m}$ Photometric Color Terms (DES PreCam Filter Set)

\begin{tabular}{lc}
\hline \hline Band & $\begin{array}{c}\text { Color Term } \\
(A B C D E)_{6}\end{array}$ \\
\hline 2015 Apr 30-May 3 & \\
$u$ & $-0.033 \pm 0.01$ \\
$g$ & $0.020 \pm 0.001$ \\
$r$ & $-0.068 \pm 0.001$ \\
$i$ & $-0.063 \pm 0.001$ \\
$z$ & $-0.026 \pm 0.002$ \\
\hline
\end{tabular}

advantage of the overlap of our multiple short exposures with large dithers to tie all of the chip data for a given field onto the same photometric zero point using an übercal technique. Although PHOTRED performs similar tasks (i.e., COMBINE and CALIB), it is on a night-by-night basis. This meant, therefore, that the custom SMASH calibration software needed to start with the instrumental PHOTRED photometry catalogs output by the ASTROM stage.
The calibration follows these steps (in pseudocode): WHILE calibrated photometry changes $>1$ mmag:

(a) The photometry is calibrated using the zero-point (ZPTERM), color (COLTERM), and extinction/airmass terms (AMTERM). This is an iterative process because of the color term. First, the source photometry is calibrated using the average photometry to construct the color (a color of zero is used on the first iteration), then the photometry is averaged per object and band. The process repeats until all changes are $<0.1 \mathrm{mmag}$. (SMASH_APPLY_PHOTTRANSEQN.PRO).

FOR all filters

1. Measure the pair-wise photometric offsets of overlapping chips (SMASH_MEASURE_MAGOFFSET.PRO).

2. Solve the relative magnitude offsets per chip using the übercal algorithm (SMASH_SOLVE_UBERCAL.PRO).

3. Determine the photometric zero-point (SMASH_SET_ZEROPOINTS. PRO).

\section{ENDFOR}

\section{ENDWHILE}

We employ a simple iterative übercal solving technique (Padmanabhan et al. 2008). After all of the pair-wise photometric offsets of overlapping chips are measured, the robust weighted-average offset of a chip relative to its overlapping neighbors is calculated and one-half of this is used as the übercal correction for this chip. The pair-wise photometric offsets are updated for these chip-wise corrections, and the procedure repeats until convergence is reached (the average relative offset change from one iteration to the next is less than $1 \%$ ). The changes become very small after only a couple of iterations. The cumulative corrections are applied to the chip-wise zero-point terms (ZPTERM) and saved in the UBERCAL_MAGOFFSET columns. The übercal technique only measures and solves for a constant magnitude offset for every chip. There is no allowance for spatial variations across the chip such as those due to variable throughput. The outer (while) loop in the calibration is used to make sure the color terms are properly taken into account.

One of three different techniques is employed to set the photometric zero point of the data depending on the observing conditions and what $0.9 \mathrm{~m}$ calibration data are available. The options in decreasing order of preference are: 
1. Photometric DECam data (ZPCALIBFLAG $=1$ ). Any DECam data taken during photometric conditions (PHOTOMETRIC $=1)$ and having good photometric transformation equations from standard star exposures $($ BADSOLN $=0)$ are used to set the photometric zero point. Any non-photometric data are tied to this via the übercal offsets.

2. Overlap with photometric DECam data (ZPCALIBFLAG = 2). A field with no photometric data itself but that overlaps a neighboring field (this happens mainly in the central LMC and SMC fields) with photometric data can be calibrated using the overlap. The median offset of bright, high-S/N overlap stars is used to set the zero point.

3. $0.9 \mathrm{~m}$ calibration data (ZPCALIBFLAG $=3$ ). If a field cannot be calibrated using the first two options and $0.9 \mathrm{~m}$ calibration data are available for the field, then it is used to determine the zero point with the stars detected in both DECam and $0.9 \mathrm{~m}$ data.

The type of zero-point calibration used for any field can be found in Table 1 as well as in the FIELD_chips file (see below) and chip table of the SMASH database.

For fields where none of these options are available, we use SMASH-Gaia color-color relations to calculate rough zero points (ZPCALIBFLAG $=4$ ). These relations were derived by cross-matching 49 of our SMASH fields with good, calibrated photometry and that lie far from both the LMC and SMC against the Gaia catalog. Bright stars were used to determine the functional relationship between $X_{\mathrm{SMASH}}-G_{\text {Gaia }}$ and a SMASH color $(g-i$ for all SMASH bands except $r-z$ for $g$ ). These relations are very tight for the redder bands $(r, i$, and $z)$ with only a scatter of $\sim 0.5 \%$ (see Figure 8), but are poorer and with a large color term for the bluer bands ( $u$ and $g$ with scatters of $\sim 6 \%$ and $\sim 1 \%$, respectively). These rough calibrations are a temporary measure. The remaining partially calibrated or uncalibrated fields will be fully calibrated once the appropriate calibration data have been obtained.

Once all of the data are calibrated, average coordinates and morphological parameters (e.g., sharp, chi) are computed (weighted averages) from multiple measurements of each object. We then produce an exposure map for the field in each band (at the pixel level) and use this to sort out non-detections (set to 99.99) from cases with no good data for an object (set to $\mathrm{NaN})$. Schlegel et al. (1998) $E(B-V)$ extinctions are also added for each object, but dereddened magnitudes are not computed. Care should be taken in using the SFD extinction values in the central regions of the MCs, because they can be unreliable there, and also for MW stars, for which they can overestimate the foreground dust. Finally, the unique objects are cross-matched with the Gaia, 2MASS, and ALLWISE catalogs.

\section{Description and Achieved Performance of Final Catalogs}

The SMASH data set includes 5809 DECam exposures with 349,046 separate chip files producing 3,992,314,414 independent source measurements of 418,642,941 unique objects (296,223,749 with multiple detections).

\subsection{Final Catalog Files}

The final catalogs consist of seven gzip-compressed binary FITS files per field:

1. FIELD_exposures.fits.gz-Information about each exposure.

2. FIELD_chips.fits.gz-Information about each chip.

3. FIELD_allsrc.fits.gz-All of the individual source measurements for this field.

4. FIELD_allobj.fits.gz-Average values for each unique object.

5. FIELD_allobj_bright.fits.gz-Bright stars from allobj used for cross-matching between fields.

6. FIELD_allobj_xmatch.fits.gz-Cross-matches between SMASH and Gaia, 2MASS, and ALLWISE.

7. FIELD_expmap.fits.gz-The "exposure" map per band.

More detailed descriptions of the catalogs can be found in the PHOTRED "README" file ${ }^{49}$ on the ftp site (see below).

\subsection{Photometric Precision}

The photometric precision of the final SMASH catalogs can be estimated by calculating the scatter in multiple independent measurements of the same object using bright stars. We measured the minimum of median-binned $(0.2 \mathrm{mag}$ bins $)$ photometric scatter values of bright stars for 126 deep and fully calibrated SMASH fields in each band (see Figure 9). The distributions indicate a precision of roughly $1.0 \%(u), 0.7 \%(\mathrm{~g})$, $0.5 \%(r), 0.8 \%(i)$, and $0.5 \%(z)$ in the SMASH photometry.

\subsection{Photometric Accuracy}

To evaluate the accuracy of the photometric calibration, we use the overlap of the fields in the LMC/SMC main-body fields that are independently calibrated. Using the scatter in their distributions of mean magnitude offsets (and accounting for the $\sqrt{2}$ because there are contributions from both fields), we obtain rough calibration accuracies of $1.3 \%(u), 1.3 \%(g), 1.0 \%(r)$, $1.2 \%(i)$, and $1.3 \%(z)$. The low scatter in the SMASH-Gaia color-color relations (especially for the redder bands) also attest to the high quality of the SMASH calibration.

\subsection{Photometric Depth}

The median $5 \sigma$ point source depths in the ugriz bands are (23.9, 24.8, 24.5, 24.2, 23.5) mag, respectively, which is $\sim 2$ mag deeper than SDSS and $\sim 1.4$ mag deeper than PanSTARRS1.

\subsection{Astrometric Performance}

The astrometric precision of the individual measurements of bright stars is $\sim 20$ mas. The precision of the average coordinates of the objects (each having 10-30 measurements) is $\sim 15$ mas and is limited by the systematics in the higher-order WCS distortion terms. The astrometric accuracy is $\sim 2$ mas per coordinate with respect to the Gaia reference frame.

\footnotetext{
${ }^{49} \mathrm{ftp}: / /$ archive.noao.edu/public/hlsp/smash/dr1/photred/README.txt
} 

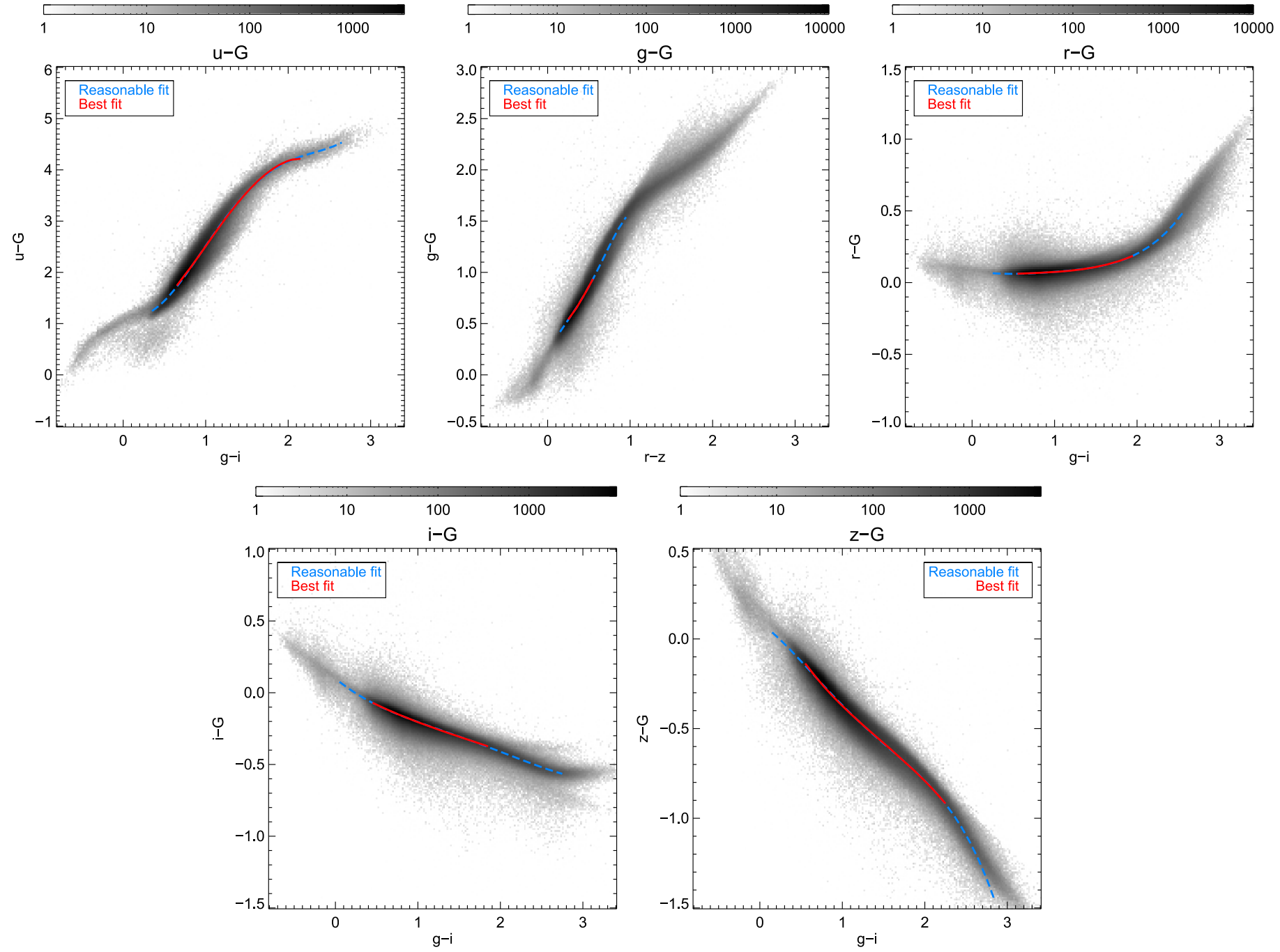

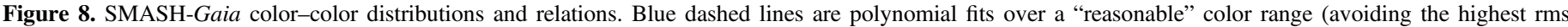

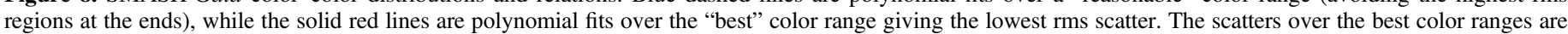
$6 \%(u), 1 \%(g), 0.2 \%(r), 0.4 \%(i)$ and $0.5 \%(z)$.

\section{First Public Data Release}

The first SMASH public data release contains $\sim 700$ million measurements of $\sim 100$ million objects in 61 deep and fully calibrated fields sampling the $\sim 2400 \mathrm{deg}^{2}$ region of the SMASH survey (blue hexagons in Figure 10). The rest of the data will be included in our second data release in 2018. The main data access is through a prototype version of the NOAO Data Lab. ${ }^{50}$ Access and exploration tools include a custom Data Discovery tool, database access to the catalog (via direct query or TAP service), an image cutout service, and a Jupyter notebook server with example notebooks for exploratory analysis. The data release page also gives extensive documentation on the SMASH survey, the observing strategy, data reduction and calibration, as well as information on the individual data products.

Images, intermediate data products, and final catalogs (in FITS binary formats) are also available through the NOAO High Level Data Products FTP site. ${ }^{51}$ The raw images as well as the CP-reduced InstCal, Resampled, and single-band

\footnotetext{
${ }^{50}$ http://datalab.noao.edu/

${ }^{51} \mathrm{ftp} / / /$ archive.noao.edu/public/hlsp/smash/dr1/
}

Stacked images are available in the raw/, instcal/, resampled/, and stacked/ directories, respectively (and grouped in nightly subdirectories). Each subdirectory has a README file that gives information about each FITS image file (e.g., exposure number, time stamp, filter, exposure time, field). The PHOTRED-ready FITS files and other associated files (PSF, photometry catalogs, logs, etc.) as well as the multiband stacks are available in the photred/ directory. The final binary FITS catalogs (as described in Section 7.1) are in the catalogs/directory. Finally, there are seven tables in the database that were populated using the FITS catalogs (but somewhat modified): field, exposure, chip, source, object, and xmatch. The "field" table includes summary information for each field. A detailed description of the database schema (tables and columns) is given on the SMASH data release Web site.

\section{Results}

SMASH is a deep, multiband photometric survey of the MCs with the goal of mapping the stellar features of these two nearby galaxies to very low surface brightness and thereby providing better understanding of their joint formation and evolution. The data in the central regions of the Clouds will 


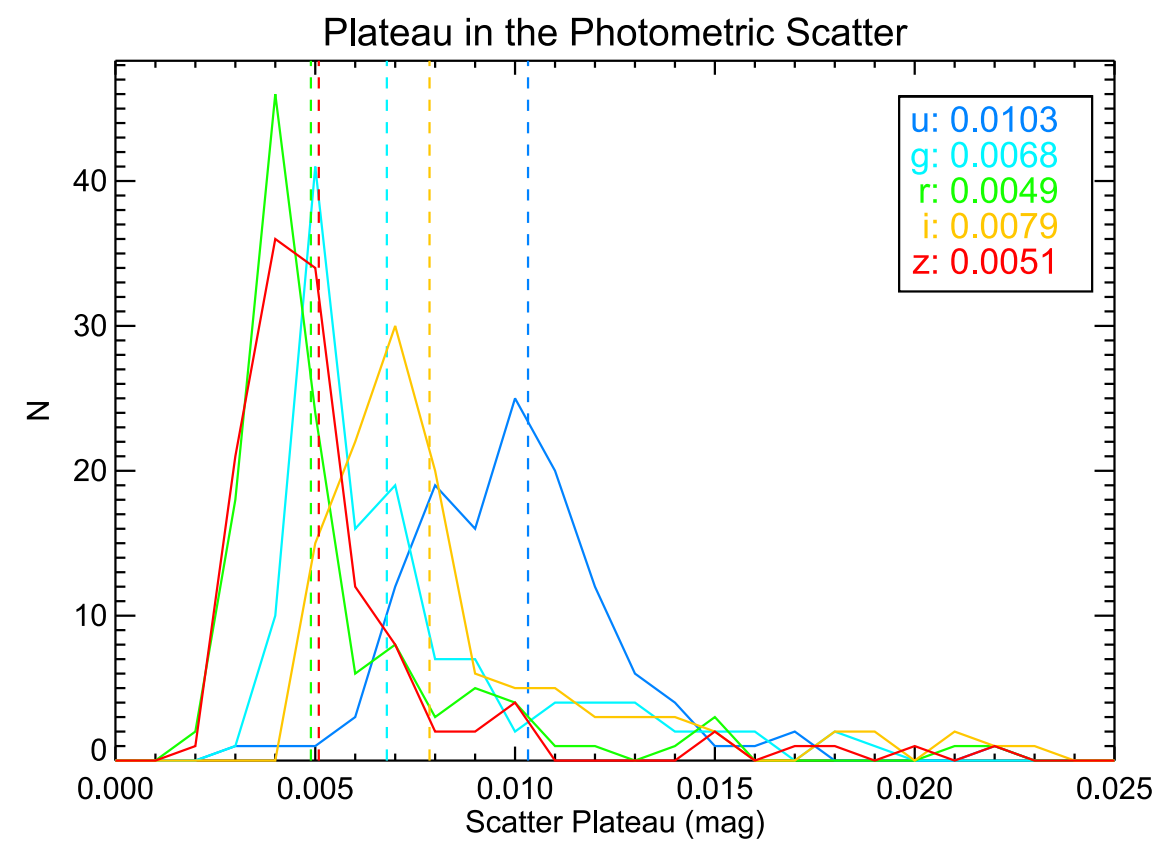

Figure 9. Distribution of the lower plateau in the photometric scatter (using multiple measurements of bright stars) in 126 calibrated SMASH fields. This is a good estimate for the photometric precision of the survey. Vertical dashed lines show the median value for each band.

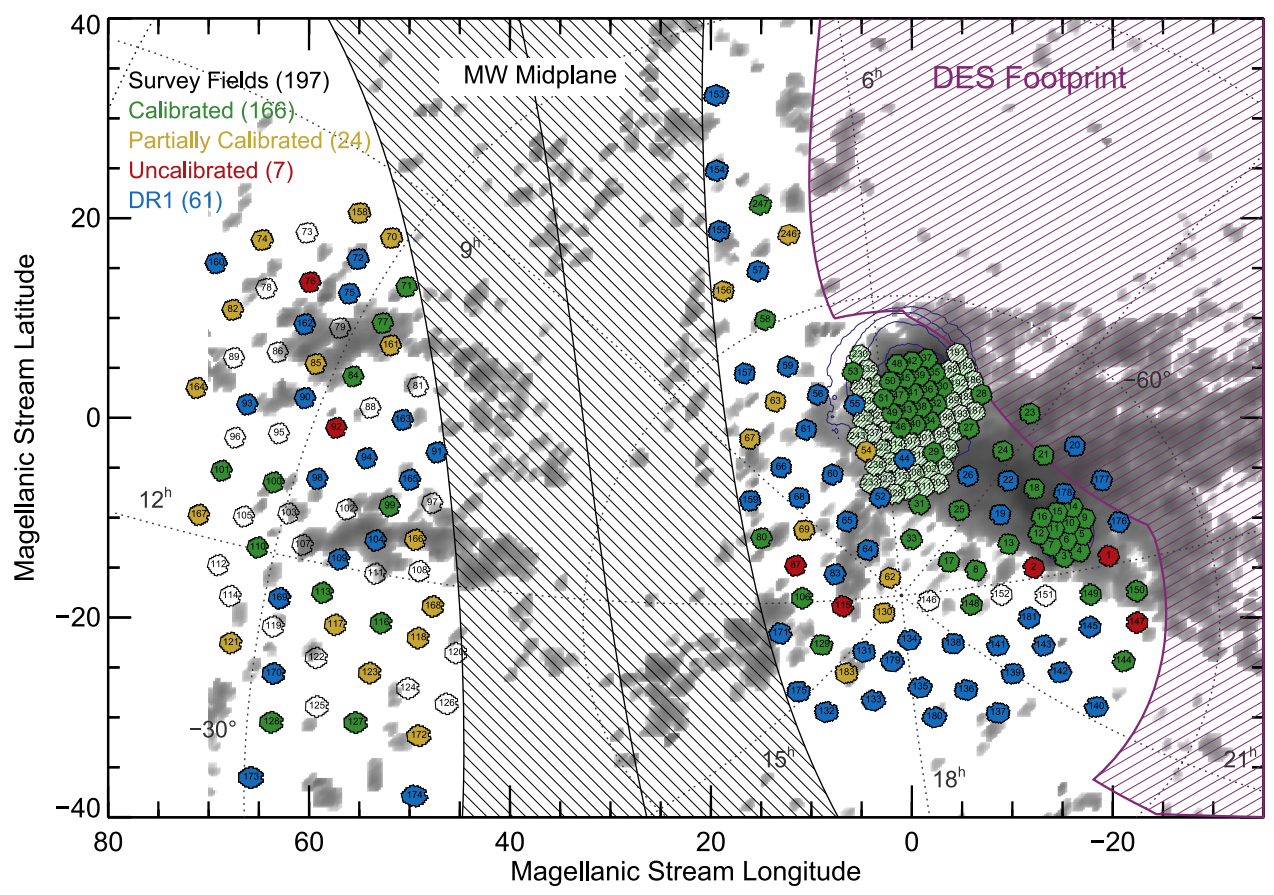

Figure 10. SMASH survey. The observed H I column density of the Magellanic Stream system is shown in grayscale (Nidever et al. 2010). Observed SMASH fields are shown as filled hexagons while unobserved SMASH fields are indicated by open black hexagons. Green (and dark blue) fields are fully calibrated (166 fields), golden are partially calibrated (some bands calibrated and some bands uncalibrated; 24 fields), and red are uncalibrated (seven fields). The green hashed hexagons are the 40 shallow LMC fields. The 61 DR1 fields are shown in dark blue (all fully calibrated). The DES footprint is represented by the purple shaded region.

also be used to obtain spatially resolved star formation histories of these galaxies to old ages. The data, spanning a region of $2400 \mathrm{deg}^{2}$, have been processed and calibrated to high fidelity with almost four billion measurements of 420 million objects in 197 fields. The data for 61 of these fields and 100 million objects are in the first public data release through the NOAO Data Lab. Figure 11 shows some example Hess diagrams of a number of our SMASH fields, which indicate the depth $(\sim 2$ mag below the oldest main-sequence turnoff in the LMC) and high quality of the SMASH photometry. In addition, Figure 12 shows multiple CMDs using all ugriz bands for Field55.

The SMASH data have already produced some exciting results. In Martin et al. (2015), we presented the discovery of a compact and faint MW satellite, Hydra II (in Field169), with morphological and stellar population properties consistent with 
Field55

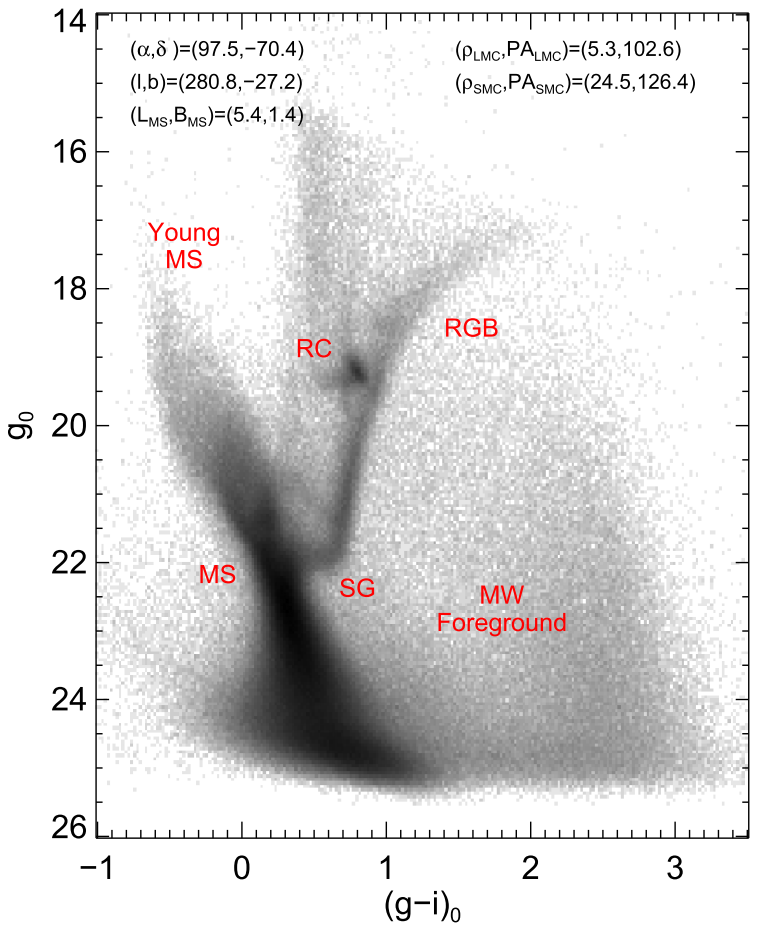

Field174

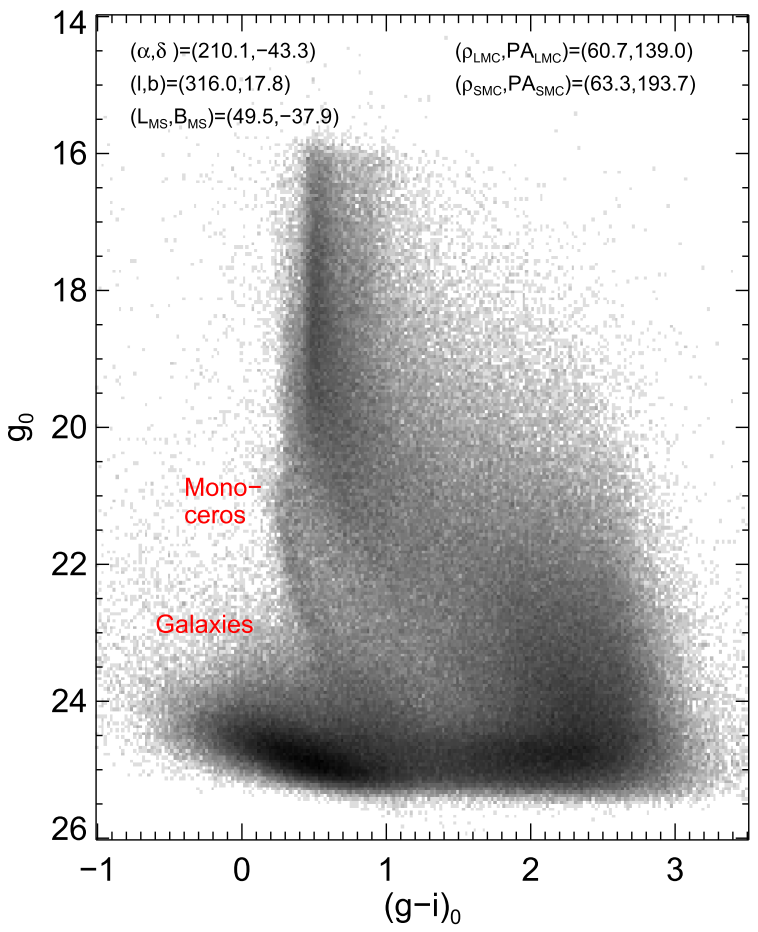

Field18

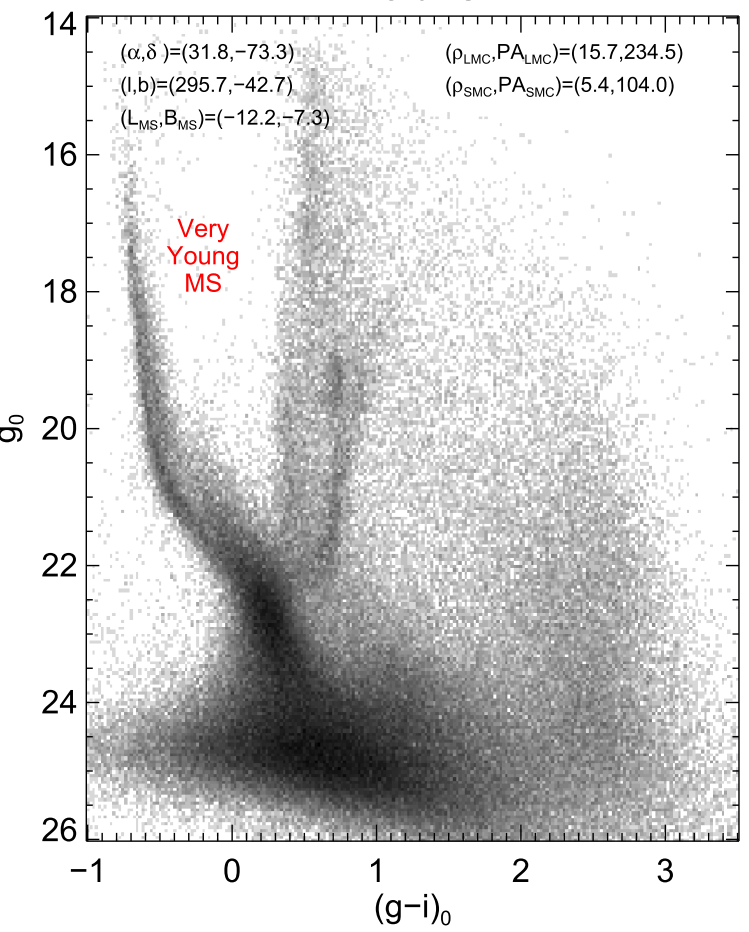

Field109

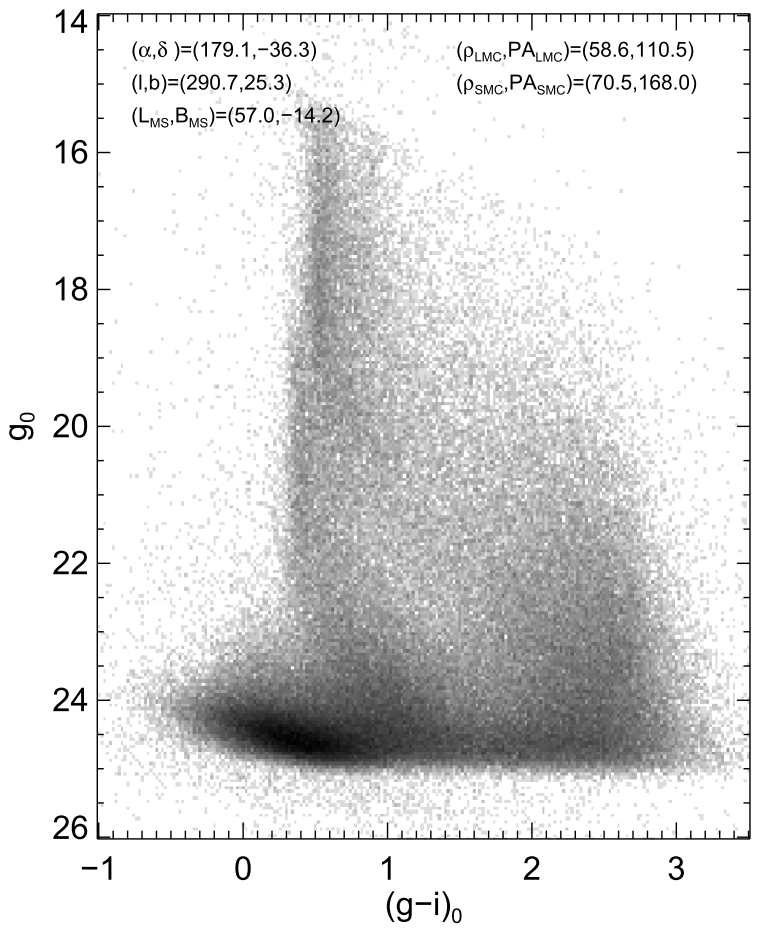

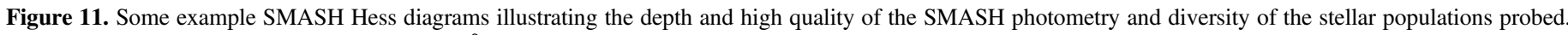

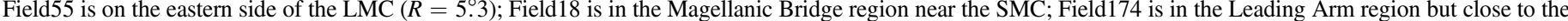

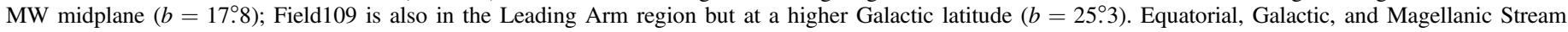

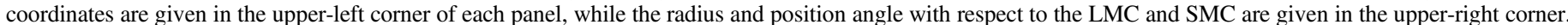

being a dwarf galaxy (also see Kirby et al. 2015). Interestingly, comparison with simulations suggests that, given Hydra II's position in the sky and distance of $\sim 140 \mathrm{kpc}$ (from BHB stars), it could be associated with the Leading Arm of the Magellanic Stream. Proper motion information, however, is needed to confirm this possibility. We obtained follow-up time-series data on Hydra II to study its variable stars. This work yielded one RR Lyrae star in Hydra II that gave a slightly larger distance of $151 \pm 8 \mathrm{kpc}$, as well as the discovery of dozens of short-period variables in the field (Vivas et al. 2016).

Further sensitive searching for overdensities in the SMASH data yielded the discovery of a compact and very faint 

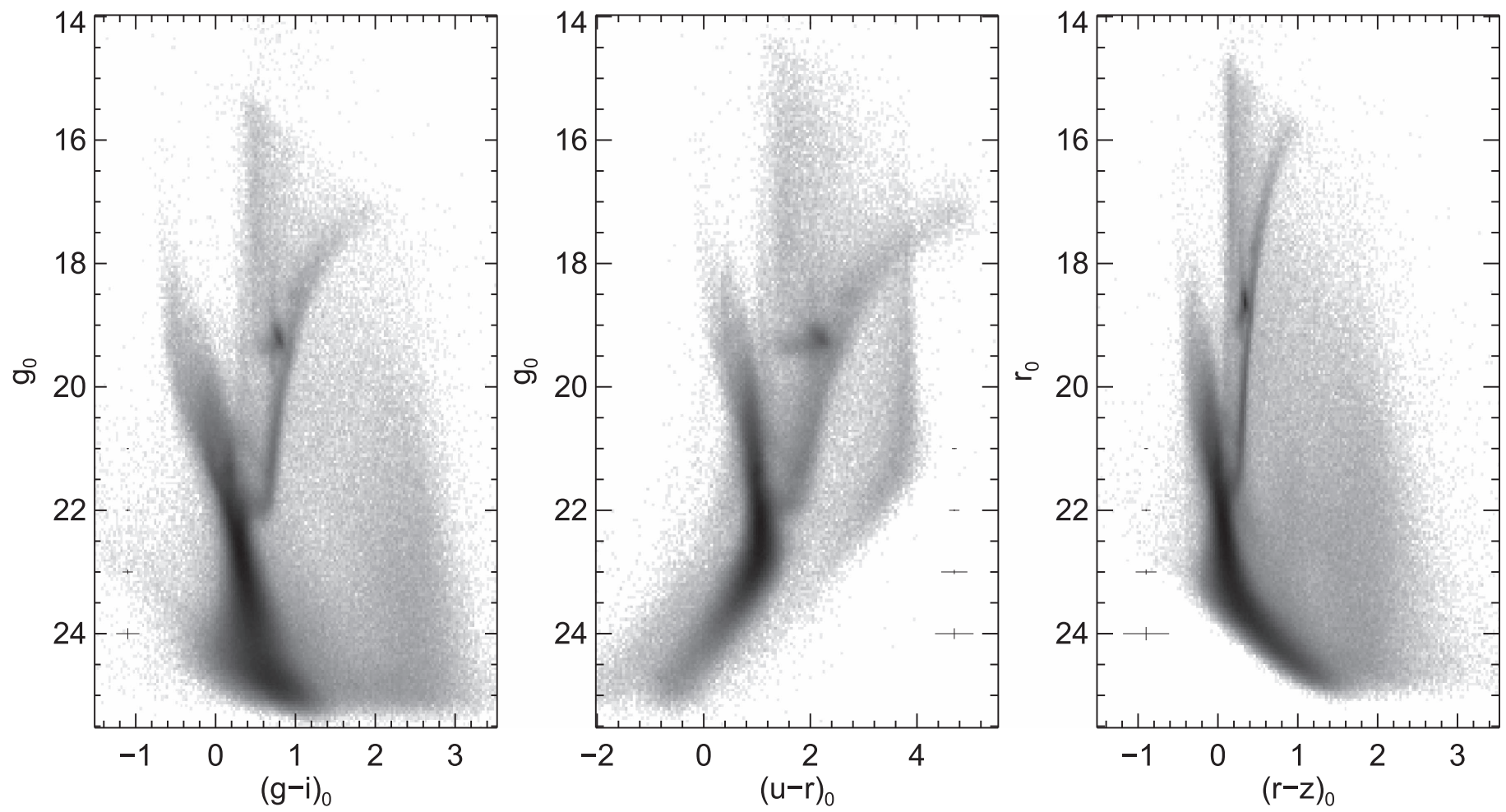

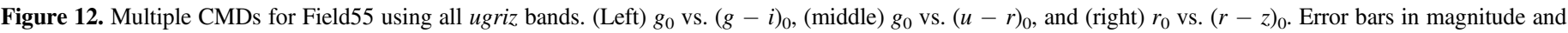
color are shown at the 21st, 22nd, 23rd, and 24th magnitude levels.

$\left(M_{V}=-1.0\right)$ stellar system (designated SMASH 1) $\sim 11^{\circ}$ away from the LMC (Martin et al. 2016). SMASH 1 is consistent with being an old globular cluster in the LMC periphery likely associated with the LMC disk and potentially on the verge of being tidally disrupted.

One of the ongoing SMASH projects is to map out the extended stellar populations of the LMC. An analysis of the Hess diagrams indicates that LMC stellar populations can be detected in SMASH data out to $21^{\circ} .1$ from the LMC center ${ }^{52}$, or $\sim 18.4 \mathrm{kpc}$, and to surface brightness levels of $\sim 33.3 \mathrm{mag} \operatorname{arcsec}^{-2}$ (D. Nidever et al. 2017, in preparation).

One of the main goals of SMASH is to use the data in the central LMC/SMC fields to derive spatially resolved star formation histories. The Hess diagram of Field55 in Figure 11 (upper left) is an example of the wealth of information in the data. This field, and other nearby ones, shows two subgiant branches, which indicate two periods of peak star formation. This was previously only seen in star formation rate diagrams from detailed star formation history modeling (Harris \& Zaritsky 2009; Meschin et al. 2014), but now is visually clear just in the Hess diagrams. Full star formation history modeling still awaits computationally intensive artificial star tests for the SMASH data, which will be a focus of ongoing SMASH processing efforts in the near future.

The deep and multiband data in the main bodies of the MCs are also very useful for detecting faint star clusters. We are in the process of developing a citizen science project (led by L.C. J.) based on the SMASH data under the Zooniverse platform, ${ }^{53}$ which currently has roughly one million users and hosts many citizen science projects in multiple scientific disciplines. The

\footnotetext{
52 We use $(\alpha, \delta)=(05: 27: 36,-69: 52: 00)$ for the LMC center and $(\alpha, \delta)$ $=(00: 52: 44,-72: 49: 42)$ for the SMC center.

53 https://www.zooniverse.org
}

project will be called "The Magellanic Project" and will be similar to the "The Andromeda Project" of HST images of M31. The citizen scientists will inspect our deep coadd ugriz images and visually identify (a) star clusters (open and globular), (b) galaxies behind the LMC/SMC main bodies, (c) and potential new dwarf galaxies of the MW or the MCs. The Web site is projected to be launched in early 2018.

The SMASH data are also very useful for studying structures in the MW halo that are unrelated to the MCs. The Hess diagram of Field174 in Figure 11 (lower-left panel), not far above the MW midplane ( $b=17.8$ ), shows a prominent stellar population at a distance of $\sim 10-20 \mathrm{kpc}$ (thin sequence with $(g-i)_{0} \sim 0.5$ and $\left.21.0 \lesssim g_{0} \lesssim 23.5\right)$. Many other fields at low Galactic latitudes show similar stellar populations that are very likely associated with the Monoceros "ring" (e.g., Slater et al. 2014). There are ongoing SMASH projects to study these and similar MW halo structures in the SMASH data.

The SMASH survey has the potential to revolutionize our understanding of the stellar populations inside and in the very outskirts of two canonical examples of dwarf galaxies, the SMC and LMC.

D.L.N. was supported by a McLaughlin Fellowship while at the University of Michigan. Y.C. acknowledges support from NSF grant AST 1655677. B.C.C. acknowledges the support of the Australian Research Council through Discovery project DP150100862. E.F.B. acknowledges support from NSF grants AST 1008342 and 1655677. E.W.O. was partially supported by NSF grant AST 1313006. T.d.B. acknowledges financial support from the ERC under Grant Agreement n. 308024. M.-R.C. acknowledges support by the German Academic Exchange Service (DAAD), from the UK's Science and Technology Facility Council (grant number ST/M001008/1), and from the the European Research Council (ERC) under the 
European Union's Horizon 2020 research and innovation programme (grant agreement No 682115). S.J. is supported by the Netherlands Organization for Scientific Research (NWO) Veni grant 639.041.131. S.R.M. acknowledges partial support from NSF grant AST 1312863. D.M.-D. acknowledges support by Sonderforschungsbereich (SFB) 881 "The Milky Way System" of the German Research Foundation (DFB), subproject A2. R.R.M. acknowledges partial support from CONICYT Anillo project ACT-1122 and project BASAL PFB-06. G.S.S. is supported by grants from NASA. Based on observations at Cerro Tololo Inter-American Observatory, National Optical Astronomy Observatory (NOAO Prop. ID: 2013A-0411 and 2013B-0440; PI: Nidever), which is operated by the Association of Universities for Research in Astronomy (AURA) under a cooperative agreement with the National Science Foundation. IRAF is distributed by the National Optical Astronomy Observatory, which is operated by the Association of Universities for Research in Astronomy (AURA) under a cooperative agreement with the National Science Foundation. This project used data obtained with the Dark Energy Camera (DECam), which was constructed by the Dark Energy Survey (DES) collaboration. Funding for the DES Projects has been provided by the U.S. Department of Energy, the U.S. National Science Foundation, the Ministry of Science and Education of Spain, the Science and Technology Facilities Council of the United Kingdom, the Higher Education Funding Council for England, the National Center for Supercomputing Applications at the University of Illinois at Urbana-Champaign, the Kavli Institute of Cosmological Physics at the University of Chicago, Center for Cosmology and Astro-Particle Physics at the Ohio State University, the Mitchell Institute for Fundamental Physics and Astronomy at Texas A\&M University, Financiadora de Estudos e Projetos, Fundação Carlos Chagas Filho de Amparo, Financiadora de Estudos e Projetos, Fundação Carlos Chagas Filho de Amparo à Pesquisa do Estado do Rio de Janeiro, Conselho Nacional de Desenvolvimento Cientfico e Tecnológico and the Ministério da Ciência, Tecnologia e Inovação, the Deutsche Forschungsgemeinschaft, and the Collaborating Institutions in the Dark Energy Survey. The Collaborating Institutions are Argonne National Laboratory, the University of California at Santa Cruz, the University of Cambridge, Centro de Investigaciones Enérgeticas, Medioambientales y Tecnológicas-Madrid, the University of Chicago, University College London, the DES-Brazil Consortium, the University of Edinburgh, the Eidgenössische Technische Hochschule (ETH) Zürich, Fermi National Accelerator Laboratory, the University of Illinois at UrbanaChampaign, the Institut de Ciències de l'Espai (IEEC/CSIC), the Institut de Física d'Altes Energies, Lawrence Berkeley National Laboratory, the Ludwig-Maximilians Universität München and the associated Excellence Cluster Universe, the University of Michigan, the National Optical Astronomy Observatory, the University of Nottingham, the Ohio State University, the University of Pennsylvania, the University of Portsmouth, SLAC National Accelerator Laboratory, Stanford University, the University of Sussex, and Texas A\&M University. This work has made use of data from the European Space Agency (ESA) mission Gaia (https://www.cosmos.esa. int/gaia), processed by the Gaia Data Processing and Analysis Consortium (DPAC; https://www.cosmos.esa.int/web/gaia/ dpac/consortium). Funding for DPAC has been provided by national institutions, in particular the institutions participating in the Gaia Multilateral Agreement. This publication makes use of data products from the Two Micron All Sky Survey, which is a joint project of the University of Massachusetts and the Infrared Processing and Analysis Center/California Institute of Technology, funded by the National Aeronautics and Space Administration and the National Science Foundation.

\section{ORCID iDs}

David L. Nidever (1D https://orcid.org/0000-0002-1793-3689 Knut Olsen (10) https://orcid.org/0000-0002-7134-8296 Alistair R. Walker (iD https://orcid.org/0000-0002-7123-8943 A. Katherina Vivas (iD https://orcid.org/0000-0003-4341-6172 Robert D. Blum (i) https://orcid.org/0000-0002-8622-4237 Yumi Choi (D) https://orcid.org/0000-0003-1680-1884 Blair C. Conn (1D https://orcid.org/0000-0001-6959-4546 Robert A. Gruendl (i) https://orcid.org/0000-0002-4588-6517 Eric F. Bell (i) https://orcid.org/0000-0002-5564-9873 Carme Gallart (iD https://orcid.org/0000-0001-6728-806X Nicolas F. Martin (i) https://orcid.org/0000-0002-1349-202X Abhijit Saha (iD https://orcid.org/0000-0002-6839-4881 Antonela Monachesi (ib https://orcid.org/0000-00032325-9616

Matteo Monelli (iD https://orcid.org/0000-0001-5292-6380

L. Clifton Johnson (i) https://orcid.org/0000-0001-6421-0953

Dennis Zaritsky (1) https://orcid.org/0000-0002-5177-727X

Guy S. Stringfellow (i) https://orcid.org/0000-0003-1479-3059 Roeland P. van der Marel (ib https://orcid.org/0000-00017827-7825

Maria-Rosa L. Cioni (1D https://orcid.org/0000-00026797-696X

Shoko Jin (1D https://orcid.org/0000-0002-4824-8430

Steven R. Majewski (iD https://orcid.org/0000-0003-

2025-3147

David Martinez-Delgado (i) https://orcid.org/0000-00033835-2231

Edouard J. Bernard (ib https://orcid.org/0000-0002-8722-225X Andrea Kunder (ib https://orcid.org/0000-0002-2808-1370 You-Hua Chu (10) https://orcid.org/0000-0003-3667-574X Cameron P. M. Bell ib https://orcid.org/0000-0003-0642-6558 Felipe Santana (iD https://orcid.org/0000-0002-4023-7649

\section{References}

Alam, S., Albareti, F. D., Allende Prieto, C., et al. 2015, ApJS, 219, 12 Bechtol, K., Drlica-Wagner, A., Balbinot, E., et al. 2015, ApJ, 807, 50 Bekki, K. 2011, MNRAS, 416, 2359

Belokurov, V., Erkal, D., Deason, A. J., et al. 2017, MNRAS, 466, 4711 Belokurov, V., Irwin, M. J., Koposov, S. E., et al. 2014, MNRAS, 441, 2124 Belokurov, V., \& Koposov, S. E. 2016, MNRAS, 456, 602 Bertin, E., \& Arnouts, S. 1996, A\&AS, 117, 393

Besla, G., Hernquist, L., \& Loeb, A. 2013, MNRAS, 428, 2342

Besla, G., Kallivayalil, N., Hernquist, L., et al. 2007, ApJ, 668, 949

Besla, G., Kallivayalil, N., Hernquist, L., et al. 2010, ApJL, 721, L97 Besla, G., Kallivayalil, N., Hernquist, L., et al. 2012, MNRAS, 421, 2109 Besla, G., Martínez-Delgado, D., van der Marel, R. P., et al. 2016, ApJ, 825, 20 Boylan-Kolchin, M., Besla, G., \& Hernquist, L. 2011, MNRAS, 414, 1560 Brüns, C., Kerp, J., Staveley-Smith, L., et al. 2005, A\&A, 432, 45B

Busha, M. T., Marshall, P. J., Wechsler, R. H., Klypin, A., \& Primack, J. 2011, ApJ, 743, 40

Chabrier, G. 2001, ApJ, 554, 1274

Chambers, K. C., Magnier, E. A., Metcalfe, N., et al. 2016, arXiv:1612.05560 Cioni, M.-R. L. 2009, A\&A, 506, 1137

Cioni, M.-R. L., Bekki, K., Girardi, L., et al. 2016, A\&A, 586, A77 Cioni, M.-R. L., Clementini, G., Girardi, L., et al. 2011, A\&A, 527, A116 Connors, T. W., Kawata, D., \& Gibson, B. K. 2006, MNRAS, 371, 108 
Connors, T. W., Kawata, D., Maddison, S. T., \& Gibson, B. K. 2004, PASA, 21,222

Dark Energy Survey Collaboration, Abbott, T., Abdalla, F. B., et al. 2016, MNRAS, 460, 1270

Deason, A. J., Belokurov, V., \& Evans, N. W. 2011, MNRAS, 416, 2903

Deason, A. J., Belokurov, V., \& Weisz, D. R. 2015a, MNRAS, 448, L77

Deason, A. J., Wetzel, A. R., Garrison-Kimmel, S., \& Belokurov, V. 2015b, MNRAS, 453, 3568

De Propris, R., Rich, R. M., Mallery, R. C., \& Howard, C. D. 2010, ApJL, 714, L249

de Vaucouleurs, G. 1955, AJ, 60, 219

Diaz, J. D., \& Bekki, K. 2012, ApJ, 750, 36

D’Onghia, E., \& Lake, G. 2008, ApJL, 686, L61

Drlica-Wagner, A., Bechtol, K., Allam, S., et al. 2016, ApJL, 833, L5

Drlica-Wagner, A., Bechtol, K., Rykoff, E. S., et al. 2015, ApJ, 813, 109

Epchtein, N., de Batz, B., Capoani, L., et al. 1997, Msngr, 87, 27

Flaugher, B., Diehl, H. T., Honscheid, K., et al. 2015, AJ, 150, 150

Fox, A. J., Wakker, B. P., Smoker, J. V., et al. 2010, ApJ, 718, 1046

Gaia Collaboration, Brown, A. G. A., Vallenari, A., et al. 2016, A\&A, 595, A2

Gallart, C., Stetson, P. B., Meschin, I. P., Pont, F., \& Hardy, E. 2008, ApJL, 682, L89

Gardiner, L. T., \& Hatzidimitriou, D. 1992, MNRAS, 257, 195

Gardiner, L. T., \& Hawkins, M. R. S. 1991, MNRAS, 251, 174

Gardiner, L. T., \& Noguchi, M. 1996, MNRAS, 278, 191

Gómez, F. A., Besla, G., Carpintero, D. D., et al. 2015, ApJ, 802, 128

Harris, J., \& Zaritsky, D. 2006, AJ, 131, 2514

Harris, J., \& Zaritsky, D. 2009, AJ, 138, 1243

Hatzidimitriou, D., \& Hawkins, M. R. S. 1989, MNRAS, 241, 667

Hatzidimitriou, D., Hawkins, M. R. S., \& Gyldenkerne, K. 1989, MNRAS, 241,645

Ivezić, Ž, Sesar, B., Jurić, M., et al. 2008, ApJ, 684, 287

Jacyszyn-Dobrzeniecka, A. M., Skowron, D. M., Mróz, P., et al. 2016, AcA, 66,149

Jacyszyn-Dobrzeniecka, A. M., Skowron, D. M., Mróz, P., et al. 2017, AcA, 67, 1 Jethwa, P., Erkal, D., \& Belokurov, V. 2016, MNRAS, 461, 2212

Kaiser, N., Burgett, W., Chambers, K., et al. 2010, Proc. SPIE, 7733, 77330E

Kallivayalil, N., van der Marel, R. P., Alcock, C., et al. 2006a, ApJ, 638, 772

Kallivayalil, N., van der Marel, R. P., \& Alcock, C. 2006b, ApJ, 652, 1213

Kallivayalil, N., van der Marel, R. P., Besla, G., Anderson, J., \& Alcock, C. 2013, ApJ, 764, 161

Kirby, E. N., Simon, J. D., \& Cohen, J. G. 2015, ApJ, 810, 56

Klypin, A., Kravtsov, A. V., Valenzuela, O., \& Prada, F. 1999, ApJ, 522, 82

Koposov, S. E., Belokurov, V., Torrealba, G., \& Evans, N. W. 2015, ApJ, 805,130

Kunkel, W. E., Demers, S., \& Irwin, M. J. 2000, AJ, 119, 2789

Kunkel, W. E., Irwin, M. J., \& Demers, S. 1997, A\&AS, 122, 463

Lynden-Bell, D. 1976, MNRAS, 174, 695

Mackey, A. D., Koposov, S. E., Erkal, D., et al. 2016, MNRAS, 459, 239

Majewski, S. R., Nidever, D. L., Muñoz, R. R., et al. 2009, in IAU Symp. 256, The Magellanic System: Stars, Gas, and Galaxies, ed. J. Th. van Loon \& J. M. Oliveira (Cambridge: Cambridge Univ. Press), 51

Martin, N. F., Jungbluth, V., Nidever, D. L., et al. 2016, ApJL, 830, L10

Martin, N. F., Nidever, D. L., Besla, G., et al. 2015, ApJL, 804, L5

Mastropietro, C., Moore, B., Mayer, L., Wadsley, J., \& Stadel, J. 2005, MNRAS, 363, 509

Mateo, M. L. 1998, ARA\&A, 36, 435

McConnachie, A. W. 2012, AJ, 144, 4

Meschin, I., Gallart, C., Aparicio, A., et al. 2014, MNRAS, 438, 1067

Miknaitis, G., Pignata, G., Rest, A., et al. 2007, ApJ, 666, 674

Minniti, D., Borissova, J., Rejkuba, M., et al. 2003, Sci, 301, 1508

Monet, D. G., Levine, S. E., Canzian, B., et al. 2003, AJ, 125, 984
Moore, B., Ghigna, S., Governato, F., et al. 1999, ApJL, 524, L19

Muller, E., Staveley-Smith, L., Zealey, W., \& Stanimirović, S. 2003, MNRAS, 339, 105

Muñoz, R. R., Majewski, S. R., Zaggia, S., et al. 2006, ApJ, 649, 201

Murai, T., \& Fujimoto, M. 1980, PASJ, 32, 581

Nidever, D. L., Majewski, S. R., \& Burton, W. B. 2008, ApJ, 679, 432

Nidever, D. L., Majewski, S. R., Butler Burton, W., \& Nigra, L. 2010, ApJ, 723, 1618

Nidever, D. L., Majewski, S. R., Muñoz, R. R., et al. 2011, ApJL, 733, L10

Nidever, D. L., Monachesi, A., Bell, E. F., et al. 2013, ApJ, 779, 145

Noël, N. E. D., \& Gallart, C. 2007, ApJL, 665, L23

Olano, C. A. 2004, A\&A, 423, 895

Olsen, K. A. G., Zaritsky, D., Blum, R. D., Boyer, M. L., \& Gordon, K. D. 2011, ApJ, 737, 29

Padmanabhan, N., Schlegel, D. J., Finkbeiner, D. P., et al. 2008, ApJ, 674, 1217

Patel, E., Besla, G., \& Sohn, S. T. 2017, MNRAS, 464, 3825

Piatti, A. E. 2017, ApJL, 834, L14

Pieres, A., Santiago, B. X., Drlica-Wagner, A., et al. 2017, MNRAS, 468 1349

Putman, M. E., Staveley-Smith, L., Freeman, K. C., Gibson, B. K., \& Barnes, D. G. 2003, ApJ, 586, 170 (P03)

Rest, A., Stubbs, C., Becker, A. C., et al. 2005, ApJ, 634, 1103

Ripepi, V., Cignoni, M., Tosi, M., et al. 2014, MNRAS, 442, 1897

Rubele, S., Girardi, L., Kerber, L., et al. 2015, MNRAS, 449, 639

Saha, A., Olszewski, E. W., Brondel, B., et al. 2010, AJ, 140, 1719

Sales, L. V., Navarro, J. F., Cooper, A. P., et al. 2011, MNRAS, 418, 648

Sales, L. V., Navarro, J. F., Kallivayalil, N., \& Frenk, C. S. 2017, MNRAS, 465, 1879

Schlafly, E. F., \& Finkbeiner, D. P. 2011, ApJ, 737, 103

Schlegel, D. J., Finkbeiner, D. P., \& Davis, M. 1998, ApJ, 500, 525

Skowron, D. M., Jacyszyn, A. M., Udalski, A., et al. 2014, ApJ, 795, 108

Skrutskie, M. F., Cutri, R. M., Stiening, R., et al. 2006, AJ, 131, 1163

Slater, C., Bell, E. F., Schlafly, E. F., et al. 2014, ApJ, 791, 9

Smith, J. A., Tucker, D. L., Kent, S., et al. 2002, AJ, 123, 2121

Soszyński, I., Udalski, A., Szymański, M. K., et al. 2016, AcA, 66, 131

Stanimirović, S., Dickey, J. M., Krčo, M., \& Brooks, A. M. 2002, ApJ, 576,773

Stanimirović, S., Staveley-Smith, L., Dickey, J. M., Sault, R. J., \& Snowden, S. L. 1999, MNRAS, 302, 417

Stanimirović, S., Staveley-Smith, L., \& Jones, P. A. 2004, ApJ, 604, 176

Staveley-Smith, L., Kim, S., Calabretta, M. R., Haynes, R. F., \& Kesteven, M. J. 2003, MNRAS, 339, 87 (S03)

Stetson, P. B. 1987, PASP, 99, 191

Stetson, P. B. 1990, PASP, 102, 932

Stetson, P. B. 1994, PASP, 106, 250

Udalski, A., Szymański, M. K., \& Szymański, G. 2015, AcA, 65, 1

Valdes, F., Gruendl, R. \& DES Project 2014, in ASP Conf. Ser. 485, Astronomical Data Analysis Software and Systems XXIII, ed. N. Manset \& P. Forshay (San Francisco, CA: ASP), 379

van der Marel, R. P. 2001, AJ, 122, 1827

van der Marel, R. P., Alves, D. R., Hardy, E., \& Suntzeff, N. B. 2002, AJ, 124, 2639

van der Marel, R. P., \& Cioni, M.-R. L. 2001, AJ, 122, 1807

Vivas, A. K., Olsen, K., Blum, R., et al. 2016, AJ, 151, 118

Walker, M. G., Mateo, M., Olszewski, E. W., et al. 2016, ApJ, 819, 53

York, D. G., Adelman, J., Anderson, J. E., Jr., et al. 2000, AJ, 120, 1579

Yoshizawa, A. M., \& Noguchi, M. 2003, MNRAS, 339, 1135

Zacharias, N., Finch, C. T., Girard, T. M., et al. 2013, AJ, 145, 44

Zaritsky, D., Harris, J., Thompson, I. B., Grebel, E. K., \& Massey, P. 2002, AJ, 123,855 